\title{
Allozyme analysis reveals a complex population structure in the southern calamary Sepioteuthis australis from Australia and New Zealand
}

\author{
Lianos Triantafillos ${ }^{1, *}$, Mark Adams ${ }^{2}$ \\ ${ }^{1}$ South Australian Aquatic Science Centre, PO Box 120, Henley Beach, South Australia 5022, Australia \\ ${ }^{2}$ South Australian Museum, Evolutionary Biology Unit, North Terrace, Adelaide, South Australia 5000, Australia
}

\begin{abstract}
Allozyme electrophoresis was used to investigate species boundaries and population genetic structure within the southern calamary Sepioteuthis australis Quoy and Gaimard. Samples collected from 17 localities around southern Australia and northern New Zealand were examined for allozyme variation at 49 loci. Of 13 polymorphic loci detected, 7 were sufficiently variable to be useful as routine genetic markers of population structure. There was little or no genetic differentiation across the entire range sampled at 5 of these 7 loci. In marked contrast, the allozyme data at 2 loci $(F d p$ and $P e p D$ ) unequivocally sorted all individuals into 1 of 3 genetic types, the geographic distributions of which exhibited a markedly non-random pattern. One type was mainly found near the western and eastern limits of the sampled area, the other type predominantly in the intervening region. Where these 2 types overlapped, a third hybrid-type was found at frequencies predicted under Hardy-Weinberg expectations. The 2 most-likely explanations for these data are: (1) there are 2 taxa within $S$. australis which produce only $\mathrm{F}_{1}$ hybrids wherever they overlap, or (2) the 2 loci $F d p$ and $P e p D$ are tightly linked and thus are not independent measures of population structure. Preliminary morphological and reproductive data support the hypothesis of 2 taxa, while mitochondrial DNAsequence data are inconclusive. It is argued that some combination of the 2 explanations may be operating. Regardless of the final outcome, the data indicate that there are a number of discrete stocks of $S$. australis in this region, a result at variance with current management perspectives on this important fishery.
\end{abstract}

KEY WORDS: Population structure $\cdot$ Allozyme $\cdot$ Southern calamary

\section{INTRODUCTION}

The southern calamary, Sepioteuthis australis Quoy and Gaimard, is a neritic species, endemic to southern Australia and northern New Zealand (Lu \& Tait 1983). It is an important component of coastal ecosystems, not only as a primary consumer of crustaceans and fishes, but also as a significant food source for numerous marine animals (Coleman 1984, Gales et al. 1993). The fishing effort directed at this species by both the commercial and recreational sectors has increased substantially over the last decade (Triantafillos 1997), re-

*E-mail: triantafillos.lianos@saugov.sa.gov.au sulting in localised depletions of stocks (Triantafillos 1998). This has raised considerable concern about the status of this resource, suggesting that continued exploitation at current levels might place the stocks at high risk of collapse. Consequently, there is an urgent need to determine the status of southern calamary stocks, and to utilise this information to help implement management strategies that will ensure the longterm sustainability of this fishery.

Like most squid species, Sepioteuthis australis is a strong swimmer and therefore has the potential for long-distance dispersal. Despite this potential, tagging studies have revealed only limited migration (Smith 1983, Triantafillos 1998). Nevertheless, such results have to be treated with caution, as recent aquarium 
trials have found that the average life expectancy of southern calamary, once tagged, is less than a few weeks (L.T. unpubl. data). No other documented information on population structure is available for the species. Clearly, more information is required for any informed management of the fishery, be it at the local or global level.

Molecular genetic studies have demonstrated a high incidence of cryptic species in squid (Augustyn \& Grant 1988, Brierley et al. 1995, Izuka et al. 1996), revealing taxa that are not routinely detectable using traditional approaches. For example, Yeatman \& Benzie (1994) identified 4 species of Photololigo using allozyme electrophoresis after a detailed morphological study had revealed only 2. Any ecological studies applied to cephalopods prior to the availability of molecular systematic evidence are suspect, since they possibly involved sample sets that were composites of 2 or more species.

Clearly, there is a need for molecular genetic data at the outset of any detailed study of stock structure. Of the many molecular techniques available, allozyme electrophoresis is still one of the most appropriate techniques for an initial assessment of both species boundaries and population structure (Avise 1994, Hillis et al. 1996). The ability to provide a rapid and comparatively inexpensive survey of a large number of individuals for a wide range of independent nuclear genetic markers makes allozyme data ideal for an examination of species boundaries. In addition, the same data can also provide some insight into the genetic structure of stocks (Ihssen et al. 1981, Ryman \& Utter 1987). Allozyme data have already proved useful for assessing intraspecific differentiation in a number of economically important cephalopod species (e.g. Katugin 1995).

With reference to Sepioteuthis australis, another systematic issue emerges. As well as the more general consideration of cryptic species, there is a need to address the status of the broadfin squid $S$. bilineata from New Zealand. A morphological study by Lu \& Tait (1983) concluded that the 2 named forms were conspecific. The validity of this conclusion can be independently assessed with allozyme data.

The present study uses a combination of molecular and morphological techniques to provide insight into 2 overlapping areas of systematic interest for the southern calamary. The first objective was to clarify the taxonomic status of Sepioteuthis throughout southern Australia and northern New Zealand. The second and more important objective was to identify the population genetic structure of Sepioteuthis in southern Australasia. Both these objectives were achieved using a variety of techniques including allozyme electrophoresis, mitochondrial DNA (mtDNA)-sequencing, and analyses of morphological characters and reproductive condition.

\section{MATERIALS AND METHODS}

Sample collection. Samples of Sepioteuthis australis Quoy and Gaimard were collected from 17 sites along the coast of southern Australia and northern New Zealand between October 1995 and January 1998 using a variety of techniques (Fig. 1, Table 1). Distances between sites ranged from 30 to $5500 \mathrm{~km}$. In addition, 3 sites were represented by replicate sample sets, 2 temporal (Pearson Island and Albany) and 1 spatial (Coles Bay; Table 1). Both the temporal replicates were collected within 16 mo of the original collection date. The spatial replicate was collected on the same day, from a location $<5 \mathrm{~km}$ from the initial site.

Soon after collection, a piece of tentacle tissue was removed from each individual and frozen in liquid nitrogen. Tissues samples were returned to the laboratory and stored at $-80^{\circ} \mathrm{C}$, pending genetic analysis.

With the exception of the Perth, Coles Bay, Hazards Beach, and Eden sample sets as well as a few individuals from Albany, Pearson Island, and New Zealand, all calamary $(\mathrm{n}=456)$ were characterized for 3 basic parameters, dorsal mantle length, gender, and sexual maturity stage. Dorsal mantle length (DML) was measured, to the nearest millimetre, from the anteriormost point of the mantle to the posterior tip. Sexual maturity and gender were assigned in accordance with the universal scale of Lipinski (1979), with Stages IV, V or VI considered mature.

The bodies of 133 calamary from Esperance, Albany, Franklin Island, Kangaroo Island and Myponga Beach were opportunistically kept for a more detailed morphological examination (Table 1). These calamary were placed in individually labelled plastic bags and frozen at $-30^{\circ} \mathrm{C}$.

Allozyme electrophoresis. The allozyme study was carried out in 2 different stages. Initially 58 calamary, comprising a small number (3 to 6) of Sepioteuthis australis from 10 sites across southern Australia (Table 1), were examined for allozyme variation at a large number of enzyme loci. An additional 10 calamary from the New Zealand sample set were also screened for all loci, once they became available near the completion of the study. The aims of this overview study were 4-fold: (1) to assess the evidence for cryptic taxa/species; (2) to identify polymorphic loci suitable for determining population structure; (3) to find electrophoretic conditions under which allozyme genotypes at these polymorphic loci could be readily assigned to individuals; (4) to set sample sizes for the second stage of analysis based on preliminary allozyme frequencies. 
Table 1. Sepioteuthis australis. Details of sample sets used in the electrophoretic study. $\mathrm{n}=$ sample size. Three replicate sample sets are presented: WA2a, WA2b and SA4a, SA4b = same place, different time; TASa, TASb $=$ same time, $\sim 5 \mathrm{~km}$ apart. ${ }^{*}$ Sites included in overview study. Numbers in parentheses = number of specimens opportunistically frozen for detailed morphological analysis. WA: Western Australia; SA: South Australia; TAS: Tasmania; NSW: New South Wales

\begin{tabular}{|c|c|c|c|c|c|c|}
\hline Sample set & Code & $\begin{array}{c}\text { Date } \\
\text { caught }\end{array}$ & $\mathrm{n}$ & $\begin{array}{l}\text { Peripheral } \\
\text { (P) }\end{array}$ & $\begin{array}{l}\text { Central } \\
\text { (C) }\end{array}$ & $\begin{array}{l}\text { Hybrid } \\
(\mathrm{H})\end{array}$ \\
\hline Perth, WA & WA1 ${ }^{*}$ & 1 Jan 96 & 50 & $50^{\mathrm{a}}$ & - & - \\
\hline Albany 96, WA & WA2a & 29 Sep 96 & 40 & $14(7)$ & $8^{a}(6)$ & $18(12)$ \\
\hline Albany 98, WA & WA2b & 26 Jan 98 & 42 & $27(27)$ & 1 (1) & $14(14)$ \\
\hline Esperance, WA & WA3 & 24 Jan 98 & 34 & $11(11)$ & $2(2)$ & $21(21)$ \\
\hline St Francis Island, SA & $\mathrm{SA} 1^{*}$ & 6 May 96 & 33 & - & 26 & 7 \\
\hline Franklin Island, SA & $\mathrm{SA} 2 *$ & 7 May 96 & 31 & - & $25(7)$ & 6 \\
\hline Flinders Island, SA & $\mathrm{SA}^{*}$ & 5 May 96 & 18 & 2 & 14 & 2 \\
\hline Pearson Island 96, SA & $\mathrm{SA} 4 \mathrm{a}^{*}$ & 4 May 96 & 27 & 2 & $14^{\mathrm{a}}$ & 11 \\
\hline Pearson Island 97, SA & SA4b & 14 May 97 & 30 & - & 25 & 5 \\
\hline Port Lincoln, SA & SA5 & 2 May 96 & 52 & - & $52^{\mathrm{a}}$ & - \\
\hline Wedge Island, SA & SA6* & 1 May 96 & 51 & 1 & 50 & - \\
\hline Kangaroo Island, SA & SA7 & 18 Apr 97 & 35 & - & $35(15)$ & - \\
\hline Glenelg, SA & SA $8^{*}$ & 30 Jan 96 & 50 & - & $49^{\mathrm{a}}$ & 1 \\
\hline Myponga Beach, SA & SA9* & 6 Feb 96 & 50 & - & $50(10)$ & - \\
\hline Coles Bay, TAS & TASa * & 9 Jan 96 & 50 & - & $49^{\mathrm{a}}$ & 1 \\
\hline Hazards Beach, TAS & TASb & 9 Jan 96 & 50 & - & 49 & 1 \\
\hline Eden, NSW & NSW1 & 4 Sep 96 & 30 & 5 & $15^{\mathrm{a}}$ & 10 \\
\hline Newcastle, NSW & NSW2* & 14 Oct 95 & 50 & $48^{\mathrm{a}}$ & - & 2 \\
\hline \multirow[t]{2}{*}{ New Zealand } & NZ & 4 Nov 98 & 34 & 21 & 1 & 12 \\
\hline & & & 757 & $181(45)$ & $465(41)$ & $111(47)$ \\
\hline
\end{tabular}

Fig. 1. Map showing collection of Sepioteuthis australis in southern Australia and New Zealand. WA: Western Australia; SA: South Australia; NSW: New South Wales; TAS: Tasmania; NZ: New Zealand

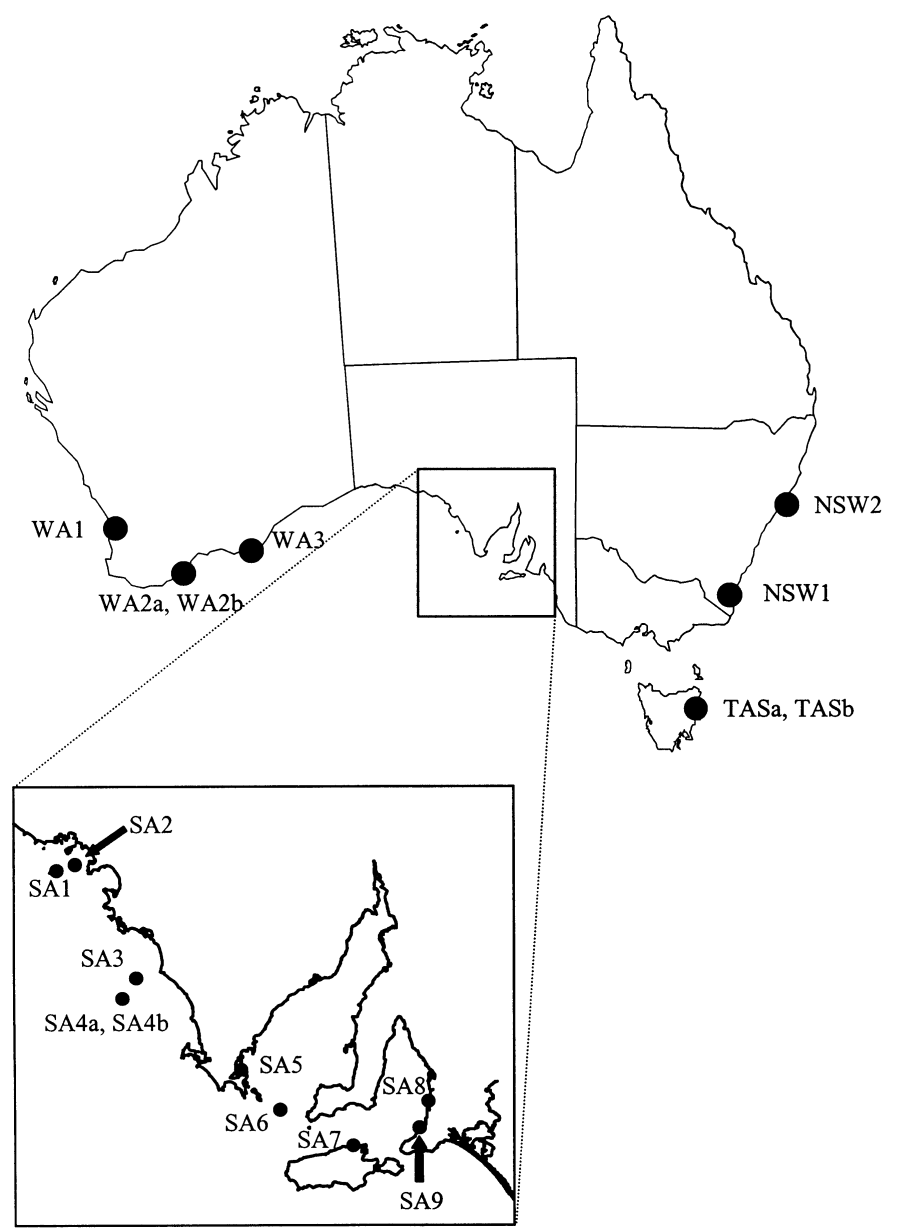

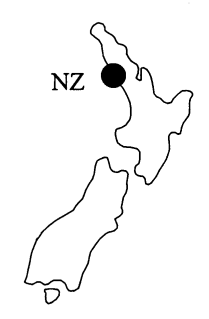


Given that enough polymorphic loci were found in the overview study to warrant an assessment of population structure (Richardson et al. 1986), a second stage of allozyme analysis was undertaken. Here, a large number of southern calamary from all 16 sites were genotyped at the polymorphic loci (Table 1, Fig. 1). Based on the overview study, the optimum sample size was set at 50 per sample set, although this was not always achieved for all sample sets. Allozyme electrophoresis was conducted according to the principles and procedures outlined in Richardson et al. Tissues were homogenized by sonication in 2 volumes of homogenizing solution (deionised water containing $0.2 \% 2$-mercaptoethanol and $0.2 \mathrm{mg} \mathrm{ml}^{-1} \mathrm{NADP}$ ). The following enzymes or non-enzymatic proteins were interpretable in the overview study: ACON (EC 4.2.1.3), ACYC (EC 3.5.1.14), ADA (EC 3.5.4.4), ADH (EC 1.1.1.1), ALD (EC 4.1.2.13), ALDH (EC 1.2.1.5), AP (EC 3.1.3.1), ARGK (EC 2.7.3.3), CA (EC 4.2.1.1), DIA (EC 1.6.99), ENOL (EC 4.2.1.11), EST (EC 3.1.1), FDP (EC 3.1.3.11), GAPD (EC 1.2.1.12), GDA (EC 3.5.4.3), GDH (EC 1.4.1.3), GLO (EC 4.4.1.5), GOT (EC 2.6.1.1), general protein, G6PD (EC 1.1.1.49), GPD (EC 1.1.1.8), GPI (EC 5.3.1.9), GPT (EC 2.6.1.2), IDH (EC 1.1.1.42), LAP (EC 3.4.11.1), LDH (EC 1.1.1.27), MDH (EC 1.1.1.37), ME (EC 1.1.1.40), MPI (EC 5.3.1.8), NDPK (EC 2.7.4.6), NP (EC 2.4.2.1), PEPA (EC 3.4.13; substrate val-leu), PEPB (EC 3.4.11; substrate leu-gly-gly), PEPD (EC 3.4.13; substrate phe-pro), PGAM (EC 5.4.2.1), 6PGD (EC 1.1.1.44), PGK (EC 2.7.2.3), PGM (EC 5.4.2.2), PK (EC 2.7.1.40), SORDH (EC 1.1.1.14) and TPI (EC 5.3.1.1). The nomenclature for referring to loci and allozymes follows Adams et al. (1987).

The allozyme data were analysed for population structure using the computer program GENEPOP Version 3.1b (Raymond \& Rousset 1995). All p values were adjusted to allow for multiple tests using the sequential Bonferroni technique (Rice 1989), applied separately for each locus and/or population combination, with an initial $\alpha$-level of 0.05. F-statistics were calculated using the program DIPLOID (Weir 1990). The overall genetic differentiation between sample sets was calculated as Nei's unbiased measure of genetic distance (Nei's $D$; Nei 1978), under the assumption that the loci monomorphic in the overview study were invariant in all sample sets. These genetic distances were depicted diagrammatically as a dendrogram in which clustering was determined by the unweighted pair-group method algorithm (UPGMA; Sokal \& Sneath 1963). The results of the allozyme study indicated the need for additional biological characterisation of selected individuals and stocks of southern calamary. This was achieved through an assessment of variability in mtDNA sequence data, external morphology, and reproductive condition. As 2 of the key genetic groups could not be re-sampled locally, the morphological characterisations were only applied a posteriori to the 133 individuals from the allozyme study that were preserved by freezing. Unfortunately, no further a posteriori examination of fertility was possible on these individuals, as the reproductive tissues deteriorate after freezing.

Mitochondrial DNA. Eight individuals of Sepioteuthis australis from 7 sites were used in a pilot study of sequence variation in portions of the mitochondrial genes COII and COIII (Table 1). These 2 genes were chosen because of the ready availability of primers shown to be successful on a wide range of squid genera (Bonnaud et al. 1997). No specimens from New Zealand were included because this sample set did not become available until near the end of the study, after the mtDNA-sequencing had been undertaken. Sequence data for both genes were obtained from the same individual in all cases, except for 1 sample from Newcastle which resisted all attempts to sequence COII; an additional individual from this site was therefore used for COII analysis. A standard phenol/chloroform extraction procedure was used to isolate DNA (Sambrook et al. 1989). Polymerase chain reaction (PCR) amplification and sequencing was carried out for an $\sim 400$ bp portion of COII and an $\sim 700$ bp portion of COIII using 2 sets of oligonucleotide primers, namely COII1 (5'ATTGCTCTGCCTTCACTACG3') and COII2 (5'CAAATTTCTGAGCATTGACC3'), plus COIII1 (5'AGCCCATGACCTTTA-ACAGG3') and COIII2 (5'GACTACATCAACAAAATGTCAGTATCA3') (Bonnaud et al. 1997). PCR reactions were carried out in $50 \mu$ reaction volumes consisting of $1 \mathrm{X}$ Taq reaction buffer (Promega), $4 \mathrm{mM}$ $\mathrm{MgCl}$, $0.8 \mathrm{mM}$ dNTP's, $0.2 \mathrm{mM}$ of each primer, 0.75 units of Taq DNA polymerase (Promega) and 50 to $100 \mathrm{ng}$ of template DNA. Amplifications were carried out on a Corbett FTS 320 Thermal Sequencer under the following conditions: 1 cycle at $94^{\circ} \mathrm{C}$ for $120 \mathrm{~s}, 48^{\circ} \mathrm{C}$ for $45 \mathrm{~s}, 72^{\circ} \mathrm{C}$ for $80 \mathrm{~s} ; 34$ cycles at $94^{\circ} \mathrm{C}$ for $45 \mathrm{~s}, 48^{\circ} \mathrm{C}$ for $45 \mathrm{~s}, 72^{\circ} \mathrm{C}$ for $60 \mathrm{~s} ; 1$ cycle at $72^{\circ} \mathrm{C}$ for $360 \mathrm{~s}$. PCR products were purified using Bresaclean, and then cycle-sequenced in $20 \mu \mathrm{l}$ reaction volumes on a Corbett FTS1 Thermal Sequencer using ABI Prism ${ }^{\odot}$ (Perkin Elmer) and procedures specified by the manufacturer. DNA sequences were determined using an $\mathrm{ABI}$ 373A autosequencer. DNA sequences were edited using SeqEd ${ }^{\mathrm{TM}}$ (Version 1.0.3, Applies Biosystem Inc., Madison, WI). Multiple seuence alignments were performed using Clustal V (Higgins et al. 1992). Aligned sequences of $912 \mathrm{bp}$ of combined COII (312 bp) and COIII (600 bp) for each individual (the Newcastle site being represented by the COII of one individual and the COIII of a second) were analysed in the phylogenetic analysis program PAUP (Version 3.1.1; Swofford 1993). Trees were generated using maximum parsimony with a heuristic search option, with all sites weighted equally. 
A COIII partial sequence of $S$. lessoniana was used as an outgroup in order to root the tree. Clade robustness was evaluated by bootstrapping over 500 pseudoreplicates. The program MEGA (Kumar et al. 1993) was used to determine the resulting amino acid sequence for each individual using the Drosophila mitochondrial genetic code, to ensure that there were no stop codons present which might indicate that a pseudogene had been amplified.

A posteriori morphological characterisation. A range of external morphological characters was initially surveyed on a handful of key specimens for the ability to distinguish the genetic groups evident from the allozyme study. Only 1 character, the number of denticles (teeth) on the largest suckers of the largest tentacles, proved likely to be informative. Consequently, this character alone was measured on all the 133 calamary available for characterization. The 3 largest sucker rings from the 2 largest tentacles were removed from each calamary and viewed under $20 \times$ magnification. Sucker teeth were counted, and the internal diameter of the suckers were measured using a graticule eyepiece and calibration slide. A general linear model (GLM) was used to test for significant differences in sucker counts between sexes, sites and genetic types using the SPSS (Subprogram of the Statistical Package for the Social Sciences).

\section{RESULTS}

\section{Allozyme variation in Sepioteuthis australis}

A total of 49 presumptive allozyme loci displayed interpretable banding patterns after histochemical staining. Of these, 13 loci displayed electrophoretic variation consistent with the existence of $\geq 2$ alleles at a Mendelian gene. Of these loci, all except 4 (Enol, Got2, $P e p B$, and $6 P g d$ ) exhibited $q>10 \%$ in at least 1 sample set, and 5 (Dia, Fdp, Np1, PepD, Pgk) were polymorphic across the range, displaying an average of 4 alleles per locus (range 2 to 8 alleles).

The comprehensive allozyme screen comprised 757 individuals from 19 sample sets (including the 2 tem-

Table 2. Sepioteuthis australis. Association between genotypes at Fdp and PepD $(p<0.00001)$. Letters in bold indicate a 'central' allele

\begin{tabular}{|c|c|c|c|}
\hline \multirow{2}{*}{$F d p$} & & \multirow{2}{*}{$\begin{array}{l}P e p D \\
\text { af, bf, cf, df, ef, eg, eh }\end{array}$} & \multirow[b]{2}{*}{ ff, fg, fh } \\
\hline & $\begin{array}{l}\mathrm{aa}, \mathrm{ab}, \mathrm{ad}, \mathrm{ae}, \mathrm{bb}, \mathrm{bc}, \mathrm{bd} \\
\mathrm{be}, \mathrm{cc}, \mathrm{cd}, \mathrm{ce}, \mathrm{dd}, \mathrm{de}, \mathrm{ee}\end{array}$ & & \\
\hline aа & 181 & - & - \\
\hline $\mathbf{a b}$ & - & 111 & - \\
\hline bb & - & - & 465 \\
\hline
\end{tabular}

poral and 1 spatial replicates), genotyped at 13 loci. A most striking outcome of this screen was complete linkage disequilibrium between certain genotypic combinations at 2 loci, Fdp and PepD (Table 2). This occurred despite there being a large number of alleles $(n=8)$ and therefore a very large number of observed genotypes $(\mathrm{n}=25)$ at $P e p D$.

With respect to individual alleles at the 2 loci, the outcome can be explained by postulating 2 'linkage' associations; the Fdp allele with any of the 5 most cathodal alleles at $P e p D$ ( $a$ to e), and the $F d p^{b}$ allele with any of the 3 more anodal PepD alleles $(f, g, h)$. No 'recombinant' genotypic pairs were found amongst the 757 individuals examined, and no other evidence of linkage disequilibrium was found for genotypes at any other pairwise combination of the 13 loci. Most importantly, there was also no evidence of deviation from Hardy-Weinberg expectations within any sample set for either $F d p$ or $P e p D$, ruling out a common cause of apparent linkage between loci, the Wahlund effect.

The above outcome is consistent with either of 2 alternative biological scenarios. Firstly, it may be that the 2 loci are very tightly linked, such that recombination occurs at less than, on average, 1/750 occasions. Alternatively, the results may reflect the presence of 2 taxa, characterized by fixed allelic differences at Fdp and $P e p D$, plus their $F_{1}$ hybrids. For convenience, we hereafter refer to these 2 putative taxa as the 'peripheral' form $\left(F d p^{\mathrm{a}}, P e p D^{\mathrm{a}, \mathrm{b}, \mathrm{c}, \mathrm{d}, \mathrm{e}}\right)$ and the 'central' form $\left(F d p^{\mathrm{b}}\right.$, $\left.P e p D^{\mathrm{f}, \mathrm{g}, \mathrm{h}}\right)$, based on their geographic distributions across southern Australasia (Table 1, Fig. 1). The allele frequencies at the 13 polymorphic loci are presented separately for each of the 3 forms (Tables 3 to 5).

How can we distinguish between these 2 very different situations, and consequently their very different implications on stock structure within southern calamary? If the former situation is valid, then no other biological differences should exist between these 3 groups except for those influenced by genes that also lie within the Fdp-PepD linkage group. Conversely, if the latter were true, one would expect to find that the 2 putative taxa display genetic differences for other characters/biological traits. Before proceeding to analyse the allozyme data, it is therefore necessary to consider the results of these other investigations.

\section{MtDNA sequence data}

Two sequences each from individuals representing 2 'peripheral' and 5 'central' sample sets, identified from their allozyme profiles, were generated in the mtDNA pilot study. Low 


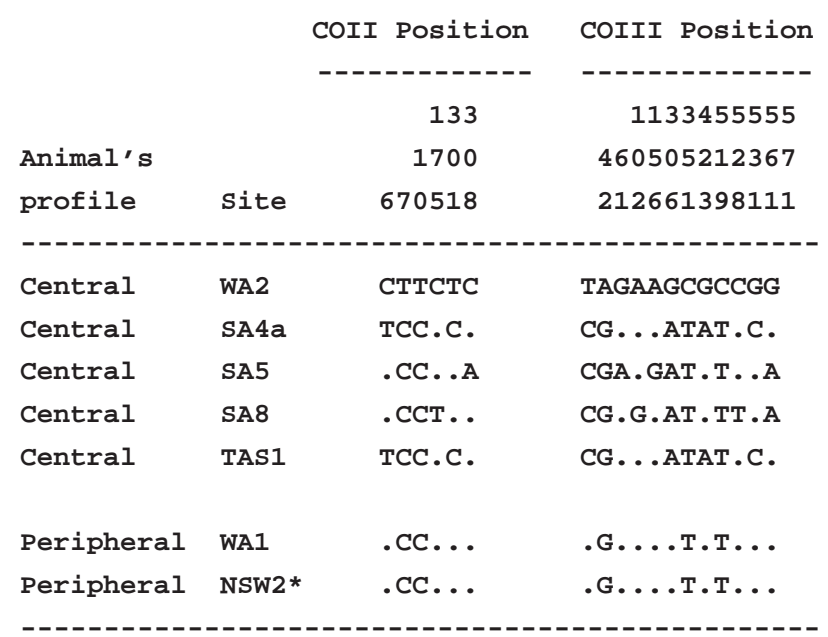

Fig. 2. Sepioteuthis australis. Summary of the variable positions found amongst 2 peripheral and 5 central calamary for the COII and COIII sequence data. COII and COIII sequences for Newcastle were derived from separate individual. Dots (.) indicate identical with the reference individual on the first

line; *additional individual used in the COII analysis levels of nucleotide diversity were detected at both protein-encoding genes. For COIII, only 12 of 600 nucleotide positions displayed variation (ignoring the outgroup Sepioteuthis lessoniana) and 7 of these involved a unique substitution in a single individual (Fig. 2). Similarly, all but 6 of the 312 nucleotide positions sequenced for COII were invariant, and only 2 of these did not involve a unique substitution in a single individual (Fig. 2). Most variants involved synonymous codon changes, with only 1 in COII (Position 308), and 2 in COIII (Positions 61 and 571), resulting in amino acid substitutions. The COIII nucleotide se-quences of S. lessoniana and $S$. australis differed at 128 to $131 / 600$ sites, equating to $\sim 6.5 \%$ (13 to $14 / 200$ ) difference in amino-acid sequence.

A phylogenetic analysis was undertaken on the combined 912 bp COII + COIII sequence data, using the COIII sequence from Sepioteuthis lessoniana as an outgroup to root the tree. One of the 2 most parsimonious trees is shown in Fig. 3 (the other displayed the same overall topology but a minor difference in 2

Table 3. Sepioteuthis australis. Allozyme frequencies in 'peripheral' calamary at the 13 polymorphic loci. All individuals were Genotype aa at Fdp. Frequencies as \% (max. 2n in parentheses)

\begin{tabular}{|c|c|c|c|c|c|c|c|c|c|c|c|}
\hline Locus & $\begin{array}{c}\text { Allele } \\
(100)\end{array}$ & $\begin{array}{l}\text { WA1 } \\
(28)\end{array}$ & $\begin{array}{c}\text { WA2a } \\
(54)\end{array}$ & $\begin{array}{c}\text { WA2b } \\
(22)\end{array}$ & $\begin{array}{c}\text { WA3 } \\
(4)\end{array}$ & $\begin{array}{c}\text { SA3 } \\
(4)\end{array}$ & $\begin{array}{c}\text { SA4a } \\
\text { (2) }\end{array}$ & $\begin{array}{l}\text { SA6 } \\
(10)\end{array}$ & $\begin{array}{c}\text { NSW1 } \\
(96)\end{array}$ & $\begin{array}{c}\text { NSW2 } \\
(42)\end{array}$ & NZ \\
\hline Dia & $\begin{array}{l}c \\
b \\
a\end{array}$ & $\begin{array}{r}70 \\
26 \\
4\end{array}$ & $\begin{array}{l}71 \\
29\end{array}$ & $\begin{array}{r}74 \\
22 \\
4\end{array}$ & $\begin{array}{l}82 \\
18\end{array}$ & $\begin{array}{l}50 \\
50\end{array}$ & $\begin{array}{l}75 \\
25\end{array}$ & 100 & $\begin{array}{l}80 \\
20\end{array}$ & $\begin{array}{r}69 \\
28 \\
3\end{array}$ & 100 \\
\hline Enol & $\begin{array}{l}c \\
b\end{array}$ & 100 & 100 & $\begin{array}{r}2 \\
98\end{array}$ & 100 & 100 & 100 & 100 & $\begin{array}{l}10 \\
90\end{array}$ & $\begin{array}{r}1 \\
99\end{array}$ & 100 \\
\hline Got1 & $\begin{array}{l}c \\
b \\
a\end{array}$ & $\begin{array}{r}1 \\
90 \\
9\end{array}$ & $\begin{array}{r}4 \\
89 \\
7\end{array}$ & $\begin{array}{r}2 \\
94 \\
4\end{array}$ & $\begin{array}{r}9 \\
91\end{array}$ & 100 & 100 & 100 & $\begin{array}{l}10 \\
90\end{array}$ & $\begin{array}{r}91 \\
9\end{array}$ & 100 \\
\hline Got2 & $b$ & 100 & 100 & 100 & 100 & 100 & 100 & 100 & 100 & 100 & 100 \\
\hline$I d h$ & $b$ & 100 & 100 & 100 & 100 & 100 & 100 & 100 & 100 & 100 & 100 \\
\hline$N p 1$ & $\begin{array}{l}e \\
d \\
c \\
b\end{array}$ & $\begin{array}{r}10 \\
7 \\
62 \\
21\end{array}$ & $\begin{array}{r}7 \\
62 \\
31\end{array}$ & $\begin{array}{r}8 \\
11 \\
72 \\
9\end{array}$ & $\begin{array}{l}10 \\
70 \\
20\end{array}$ & $\begin{array}{l}25 \\
50 \\
25\end{array}$ & $\begin{array}{l}25 \\
75\end{array}$ & 100 & 100 & $\begin{array}{r}2 \\
6 \\
79 \\
13\end{array}$ & $\begin{array}{l}13 \\
75\end{array}$ \\
\hline$N p 2$ & $\begin{array}{l}c \\
b \\
a\end{array}$ & $\begin{array}{r}1 \\
99\end{array}$ & $\begin{array}{r}7 \\
93\end{array}$ & $\begin{array}{r}2 \\
98\end{array}$ & 100 & 100 & 100 & 100 & 100 & $\begin{array}{r}99 \\
1\end{array}$ & 100 \\
\hline РерB & $\begin{array}{l}b \\
a\end{array}$ & $\begin{array}{r}99 \\
1\end{array}$ & 100 & 100 & 100 & 100 & 100 & 100 & 100 & $\begin{array}{r}99 \\
1\end{array}$ & 100 \\
\hline PepD & $\begin{array}{l}e \\
d \\
c \\
b \\
a\end{array}$ & $\begin{array}{r}33 \\
23 \\
22 \\
17 \\
5\end{array}$ & $\begin{array}{r}32 \\
11 \\
24 \\
29 \\
4\end{array}$ & $\begin{array}{r}26 \\
22 \\
35 \\
13 \\
4\end{array}$ & $\begin{array}{r}32 \\
27 \\
36 \\
5\end{array}$ & $\begin{array}{l}25 \\
50\end{array}$ & $\begin{array}{l}50 \\
25 \\
25\end{array}$ & $\begin{array}{l}50 \\
50\end{array}$ & 100 & 99 & 100 \\
\hline $6 P g d$ & $\begin{array}{l}b \\
a\end{array}$ & $\begin{array}{r}99 \\
1\end{array}$ & 100 & 100 & 100 & 100 & 100 & 100 & 100 & 100 & 100 \\
\hline Pgm & $\begin{array}{l}e \\
d \\
c \\
b \\
a\end{array}$ & $\begin{array}{r}1 \\
97 \\
1 \\
1\end{array}$ & $\begin{array}{r}96 \\
4\end{array}$ & $\begin{array}{r}2 \\
96\end{array}$ & 100 & 100 & 100 & 100 & $\begin{array}{l}80 \\
10 \\
10\end{array}$ & $\begin{array}{r}97 \\
2 \\
1\end{array}$ & 100 \\
\hline Pgk & $\begin{array}{l}b \\
a\end{array}$ & $\begin{array}{l}73 \\
27\end{array}$ & $\begin{array}{l}64 \\
36\end{array}$ & $\begin{array}{l}74 \\
26\end{array}$ & $\begin{array}{l}77 \\
23\end{array}$ & $\begin{array}{l}75 \\
25\end{array}$ & $\begin{array}{l}50 \\
50\end{array}$ & 100 & $\begin{array}{l}60 \\
40\end{array}$ & $\begin{array}{l}82 \\
18\end{array}$ & 100 \\
\hline
\end{tabular}


Table 4. Sepioteuthis australis. Allozyme frequencies in 'central' calamary at the 13 polymorphic loci. All individuals were Genotype $b b$ at Fdp. Frequencies as \% (max. 2n in parentheses)

\begin{tabular}{|c|c|c|c|c|c|c|c|c|c|c|c|c|c|c|c|c|c|c|}
\hline Locus & Allele & $\begin{array}{l}\text { WA2a } \\
(16)\end{array}$ & $\begin{array}{c}\text { WA2b } \\
(2)\end{array}$ & $\begin{array}{l}\text { WA3 } \\
(4)\end{array}$ & $\begin{array}{l}\text { SA1 } \\
(52)\end{array}$ & $\begin{array}{l}\text { SA2 } \\
(50)\end{array}$ & $\begin{array}{l}\text { SA3 } \\
(28)\end{array}$ & $\begin{array}{c}\text { SA4a } \\
(28)\end{array}$ & $\begin{array}{c}\text { SA4b } \\
(50)\end{array}$ & $\begin{array}{l}\text { SA5 } \\
(104)\end{array}$ & $\begin{array}{l}\text { SA6 } \\
(100)\end{array}$ & $\begin{array}{l}\text { SA7 } \\
(70)\end{array}$ & $\begin{array}{l}\text { SA9 } \\
(100)\end{array}$ & $\begin{array}{l}\text { SA8 } \\
(98)\end{array}$ & $\begin{array}{c}\text { TASa } \\
(98)\end{array}$ & $\begin{array}{c}\text { TASb } \\
(98)\end{array}$ & $\begin{array}{c}\text { NSW1 } \\
(30)\end{array}$ & $\begin{array}{l}\text { NZ } \\
(2)\end{array}$ \\
\hline Dia & $\begin{array}{l}c \\
b \\
a\end{array}$ & $\begin{array}{l}68 \\
13 \\
19\end{array}$ & $\begin{array}{l}50 \\
50\end{array}$ & 100 & $\begin{array}{r}61 \\
35 \\
4\end{array}$ & $\begin{array}{r}56 \\
42 \\
2\end{array}$ & $\begin{array}{l}61 \\
39\end{array}$ & $\begin{array}{l}64 \\
36\end{array}$ & $\begin{array}{r}68 \\
28 \\
4\end{array}$ & $\begin{array}{r}63 \\
31 \\
6\end{array}$ & $\begin{array}{r}58 \\
37 \\
5\end{array}$ & $\begin{array}{r}54 \\
37 \\
9\end{array}$ & $\begin{array}{r}67 \\
27 \\
6\end{array}$ & $\begin{array}{l}60 \\
28 \\
12\end{array}$ & $\begin{array}{r}57 \\
38 \\
5\end{array}$ & $\begin{array}{r}63 \\
33 \\
4\end{array}$ & $\begin{array}{l}73 \\
27\end{array}$ & 100 \\
\hline Enol & $\begin{array}{l}c \\
b \\
a\end{array}$ & 100 & 100 & 100 & $\begin{array}{r}4 \\
96\end{array}$ & $\begin{array}{r}2 \\
98\end{array}$ & $\begin{array}{r}4 \\
96\end{array}$ & 100 & $\begin{array}{r}2 \\
98\end{array}$ & $\begin{array}{r}3 \\
96 \\
1\end{array}$ & $\begin{array}{r}4 \\
96\end{array}$ & $\begin{array}{r}3 \\
97\end{array}$ & $\begin{array}{r}2 \\
95 \\
3\end{array}$ & $\begin{array}{r}1 \\
99\end{array}$ & $\begin{array}{r}4 \\
96\end{array}$ & $\begin{array}{r}1 \\
99\end{array}$ & 100 & 100 \\
\hline Got1 & $\begin{array}{l}c \\
b \\
a\end{array}$ & $\begin{array}{r}94 \\
6\end{array}$ & 100 & 100 & $\begin{array}{r}98 \\
2\end{array}$ & $\begin{array}{r}94 \\
6\end{array}$ & $\begin{array}{r}96 \\
4\end{array}$ & $\begin{array}{r}96 \\
4\end{array}$ & $\begin{array}{r}98 \\
2\end{array}$ & $\begin{array}{r}1 \\
95 \\
4\end{array}$ & $\begin{array}{r}94 \\
6\end{array}$ & $\begin{array}{r}91 \\
9\end{array}$ & $\begin{array}{r}2 \\
94 \\
4\end{array}$ & $\begin{array}{r}2 \\
91 \\
7\end{array}$ & $\begin{array}{l}89 \\
11\end{array}$ & $\begin{array}{r}2 \\
88 \\
10\end{array}$ & $\begin{array}{r}93 \\
7\end{array}$ & 100 \\
\hline Got2 & $\begin{array}{l}d \\
c \\
b \\
a\end{array}$ & 100 & 100 & 100 & 100 & 100 & 100 & $\begin{array}{r}96 \\
4\end{array}$ & 100 & 100 & 100 & $\begin{array}{r}97 \\
3\end{array}$ & 100 & 100 & $\begin{array}{r}1 \\
1 \\
98\end{array}$ & 100 & 100 & 100 \\
\hline$I d h$ & $\begin{array}{l}b \\
a\end{array}$ & 100 & 100 & 100 & 100 & 100 & 100 & 100 & 100 & $\begin{array}{r}99 \\
1\end{array}$ & 100 & 100 & 100 & 100 & 100 & 100 & 100 & 100 \\
\hline Np1 & $\begin{array}{l}e \\
d \\
c \\
b \\
a\end{array}$ & $\begin{array}{r}7 \\
43 \\
43 \\
7\end{array}$ & $\begin{array}{l}50 \\
50\end{array}$ & 100 & $\begin{array}{r}4 \\
6 \\
71 \\
19\end{array}$ & $\begin{array}{r}6 \\
75 \\
19\end{array}$ & $\begin{array}{r}11 \\
7 \\
71 \\
11\end{array}$ & $\begin{array}{r}7 \\
4 \\
71 \\
18\end{array}$ & $\begin{array}{r}8 \\
4 \\
69 \\
19\end{array}$ & $\begin{array}{r}8 \\
5 \\
73 \\
13 \\
1\end{array}$ & $\begin{array}{r}11 \\
7 \\
60 \\
21 \\
1\end{array}$ & $\begin{array}{r}5 \\
4 \\
73 \\
18\end{array}$ & $\begin{array}{r}7 \\
7 \\
62 \\
24\end{array}$ & $\begin{array}{r}7 \\
5 \\
77 \\
11\end{array}$ & $\begin{array}{r}6 \\
8 \\
60 \\
26\end{array}$ & $\begin{array}{r}7 \\
7 \\
72 \\
14\end{array}$ & 100 & $\begin{array}{l}50 \\
50\end{array}$ \\
\hline$N p 2$ & $\begin{array}{l}c \\
b \\
a\end{array}$ & 100 & 100 & 100 & 100 & 100 & $\begin{array}{r}96 \\
4\end{array}$ & 100 & 100 & 100 & $\begin{array}{r}1 \\
99\end{array}$ & 100 & $\begin{array}{r}1 \\
99\end{array}$ & $\begin{array}{r}99 \\
1\end{array}$ & 100 & $\begin{array}{r}99 \\
1\end{array}$ & 100 & 100 \\
\hline РерВ & $\begin{array}{l}c \\
b \\
a\end{array}$ & 100 & 100 & 100 & 100 & 100 & 100 & $\begin{array}{r}4 \\
96\end{array}$ & 100 & 100 & $\begin{array}{r}99 \\
1\end{array}$ & 100 & $\begin{array}{r}1 \\
97 \\
2\end{array}$ & 100 & $\begin{array}{r}1 \\
98 \\
1\end{array}$ & 100 & 100 & 100 \\
\hline PepD & $\begin{array}{l}h \\
g \\
f\end{array}$ & 100 & $\begin{array}{l}50 \\
50\end{array}$ & $\begin{array}{l}25 \\
75\end{array}$ & 100 & $\begin{array}{r}4 \\
96\end{array}$ & $\begin{array}{r}4 \\
96\end{array}$ & 100 & $\begin{array}{r}8 \\
92\end{array}$ & $\begin{array}{r}1 \\
99\end{array}$ & $\begin{array}{r}1 \\
99\end{array}$ & $\begin{array}{r}1 \\
99\end{array}$ & $\begin{array}{r}1 \\
99\end{array}$ & $\begin{array}{r}2 \\
2 \\
96\end{array}$ & $\begin{array}{r}1 \\
99\end{array}$ & $\begin{array}{r}1 \\
1 \\
98\end{array}$ & 100 & 100 \\
\hline $6 P g d$ & $\begin{array}{l}c \\
b \\
a\end{array}$ & 100 & 100 & 100 & 100 & 100 & 100 & 100 & $\begin{array}{r}2 \\
98\end{array}$ & $\begin{array}{r}1 \\
99\end{array}$ & 100 & 100 & 100 & $\begin{array}{r}1 \\
99\end{array}$ & $\begin{array}{r}97 \\
3\end{array}$ & $\begin{array}{r}1 \\
98 \\
1\end{array}$ & 100 & 100 \\
\hline Pgm & $\begin{array}{l}d \\
c \\
b \\
a\end{array}$ & 100 & 100 & 100 & $\begin{array}{r}96 \\
4\end{array}$ & 96 & $\begin{array}{r}96 \\
4\end{array}$ & 100 & $\begin{array}{r}94 \\
6\end{array}$ & $\begin{array}{r}98 \\
1 \\
1\end{array}$ & 100 & $\begin{array}{r}96 \\
3 \\
1\end{array}$ & 99 & $\begin{array}{r}98 \\
2\end{array}$ & $\begin{array}{r}99 \\
1\end{array}$ & $\begin{array}{r}97 \\
1 \\
1 \\
1\end{array}$ & 100 & 100 \\
\hline Pgk & $\begin{array}{l}b \\
a\end{array}$ & $\begin{array}{l}81 \\
19\end{array}$ & 100 & $\begin{array}{l}75 \\
25\end{array}$ & $\begin{array}{l}73 \\
27\end{array}$ & $\begin{array}{l}74 \\
26\end{array}$ & $\begin{array}{l}79 \\
21\end{array}$ & $\begin{array}{l}61 \\
39\end{array}$ & $\begin{array}{l}70 \\
30\end{array}$ & $\begin{array}{l}68 \\
32\end{array}$ & $\begin{array}{l}67 \\
33\end{array}$ & $\begin{array}{l}63 \\
37\end{array}$ & $\begin{array}{l}70 \\
30\end{array}$ & $\begin{array}{l}67 \\
33\end{array}$ & $\begin{array}{l}70 \\
30\end{array}$ & $\begin{array}{l}73 \\
27\end{array}$ & $\begin{array}{l}77 \\
23\end{array}$ & 100 \\
\hline
\end{tabular}

branch lengths). Of the 4 clades identified, 3 have bootstrap values of $\geq 60 \%$, but neither the 'peripheral' nor 'central' haplotypes are strictly monophyletic with respect to each other. While the 2 'peripheral' specimens display identical sequences and form a monophyletic cluster, they also cluster with one of the 'central' specimens (from Albany, Western Australia, a region of pronounced overlap between the 2 putative taxa). As there is no clear support for the genetic distinctiveness of the 2 taxa, and given the low levels of nucleotide diversity and the additional expense involved, no further sequencing was undertaken.

\section{Field examination of specimens a priori}

Significant differences between the 3 genetic groups were evident for all 3 parameters (gender, stage of sexual maturity, and age-adjusted DML: see Table 6) rou- tinely determined on most calamary. The proportion of females amongst hybrid calamary $(18.2 \%)$ was significantly less than in peripheral $(38.3 \%)$ or central $(25.7 \%)$ forms ( $p=0.0155$ for $3 \times 2$ contingency table; $p=0.0555$ for central vs peripheral only). Simple comparisons of DML between groups are not appropriate without first correcting for age-related differences between individuals. This analysis will be presented in detail elsewhere (L.T. unpubl. data). Considering only sexually mature individuals, both female and male hybrids were significantly larger than their peripheral or central counterparts (Table 6; $p<0.001$ ), a trend that exists with all hybrid age classes (L.T. unpubl. data).

One of the critical pieces of evidence needed to distinguish between the competing hypotheses of simple linkage and 2 hybridizing taxa is the reproductive potential of hybrids. In the absence of any linkage, the allozyme data indicate hybrids would need to be 
Table 5. Sepioteuthis australis. Allozyme frequencies in 'hybrid' calamary at the 13 polymorphic loci. All individuals were Genotype $a b$ at Fdp. Frequencies as \% (max. 2n in parentheses)

\begin{tabular}{|c|c|c|c|c|c|c|c|c|c|c|c|c|c|c|c|}
\hline Locus & $\begin{array}{c}\text { Allele } \\
(36)\end{array}$ & $\begin{array}{c}\text { WA2a } \\
(28)\end{array}$ & $\begin{array}{c}\text { WA2b } \\
(42)\end{array}$ & $\begin{array}{l}\text { WA3 } \\
(14)\end{array}$ & $\begin{array}{l}\text { SA1 } \\
(12)\end{array}$ & $\begin{array}{c}\text { SA2 } \\
(4)\end{array}$ & $\begin{array}{c}\text { SA3 } \\
22)\end{array}$ & $\begin{array}{c}\text { SA4a } \\
(10)\end{array}$ & $\begin{array}{c}\text { SA4b } \\
(2)\end{array}$ & $\begin{array}{c}\text { SA8 } \\
(2)\end{array}$ & $\begin{array}{c}\text { TASa } \\
(2)\end{array}$ & $\begin{array}{c}\text { TASb } \\
(20)\end{array}$ & $\begin{array}{c}\text { NSW1 } \\
\text { (4) }\end{array}$ & $\begin{array}{c}\text { NSW2 } \\
(24)\end{array}$ & NZ \\
\hline \multirow[t]{3}{*}{ Dia } & $C$ & 67 & 71 & 69 & 79 & 50 & 50 & 64 & 40 & & & 50 & 50 & 50 & \\
\hline & $b$ & 19 & 25 & 31 & 14 & 50 & 50 & 36 & 50 & 100 & 100 & & 45 & 50 & 100 \\
\hline & $a$ & 14 & 4 & & 7 & & & & 10 & & & 50 & 5 & & \\
\hline \multirow[t]{2}{*}{ Enol } & $C$ & & & & & & & 9 & & & & & 5 & & \\
\hline & $b$ & 100 & 100 & 100 & 100 & 100 & 100 & 91 & 100 & 100 & 100 & 100 & 95 & 100 & 100 \\
\hline \multirow[t]{3}{*}{ Got1 } & $C$ & & & & & & & 5 & & & & 50 & & & \\
\hline & $b$ & 97 & 100 & 98 & 93 & 92 & 75 & 86 & 80 & 100 & 100 & 50 & 95 & 100 & 100 \\
\hline & $a$ & 3 & & 2 & 7 & 8 & 25 & 9 & 20 & & & & 5 & & \\
\hline \multirow[t]{2}{*}{ Got2 } & $C$ & & & & 7 & & & & & & & & & & \\
\hline & $b$ & 100 & 100 & 100 & 93 & 100 & 100 & 100 & 100 & 100 & 100 & 100 & 100 & 100 & 100 \\
\hline \multirow[t]{2}{*}{$I d h$} & $C$ & & & & & & & 5 & & & & & & & \\
\hline & $b$ & 100 & 100 & 100 & 100 & 100 & 100 & 95 & 100 & 100 & 100 & 100 & 100 & 100 & 100 \\
\hline \multirow[t]{4}{*}{$N p 1$} & $e$ & 12 & 8 & 10 & 7 & & & 5 & & & 50 & 50 & $?$ & & \\
\hline & $d$ & & & & & 17 & & 5 & 20 & & 50 & & $?$ & 25 & \\
\hline & $C$ & 69 & 88 & 77 & 86 & 75 & 50 & 75 & 80 & 100 & & 50 & $?$ & 75 & 12 \\
\hline & $b$ & 19 & 4 & 13 & 7 & 8 & 50 & 15 & & & & & $?$ & & 88 \\
\hline \multirow[t]{2}{*}{$N p 2$} & $C$ & & 7 & & & & & & & & & & & & 4 \\
\hline & $\begin{array}{l}b \\
a\end{array}$ & 100 & 93 & $\begin{array}{r}98 \\
2\end{array}$ & 100 & 100 & 100 & 100 & 100 & 100 & 100 & 100 & 100 & 100 & 96 \\
\hline РepB & $b$ & 100 & 100 & 100 & 100 & 100 & 100 & 100 & 100 & 100 & 100 & 100 & 100 & 100 & 100 \\
\hline \multirow[t]{8}{*}{$P e p D$} & $h$ & 3 & & & & & & & & & & & & & \\
\hline & $g$ & 3 & & & 7 & & & & & & & & & & \\
\hline & $f$ & 44 & 50 & 50 & 43 & 50 & 50 & 50 & 50 & 50 & 50 & 50 & 50 & 50 & 50 \\
\hline & $e$ & 28 & 15 & 9 & 15 & 8 & & 9 & 10 & 50 & 50 & 50 & 45 & 50 & 50 \\
\hline & $d$ & 8 & 14 & 10 & 14 & 25 & & 4 & 20 & & & & & & \\
\hline & $C$ & 3 & 21 & 17 & 7 & 17 & 25 & & 20 & & & & 5 & & \\
\hline & $b$ & 11 & & 2 & 7 & & & 32 & & & & & & & \\
\hline & $a$ & & & 12 & 7 & & 25 & 5 & & & & & & & \\
\hline \multirow[t]{2}{*}{$6 P g d$} & $C$ & & & & & & & & & & & & & 25 & \\
\hline & $\begin{array}{l}b \\
a\end{array}$ & $\begin{array}{r}97 \\
3\end{array}$ & 100 & 100 & 100 & 100 & 100 & 100 & 100 & 100 & 100 & 100 & $\begin{array}{r}95 \\
5\end{array}$ & 75 & 100 \\
\hline \multirow[t]{2}{*}{$P g m$} & $e$ & & & 2 & & & & & & & & & & & \\
\hline & $d$ & 100 & 100 & 98 & 100 & 100 & 100 & 100 & 100 & 100 & 100 & 100 & 100 & 100 & 100 \\
\hline \multirow[t]{2}{*}{$P g k$} & $b$ & 61 & 75 & 83 & 57 & 67 & 50 & 77 & 70 & 100 & 50 & 100 & 65 & 75 & 100 \\
\hline & $a$ & 39 & 25 & 17 & 43 & 33 & 50 & 23 & 30 & & 50 & & 35 & 25 & \\
\hline
\end{tabular}

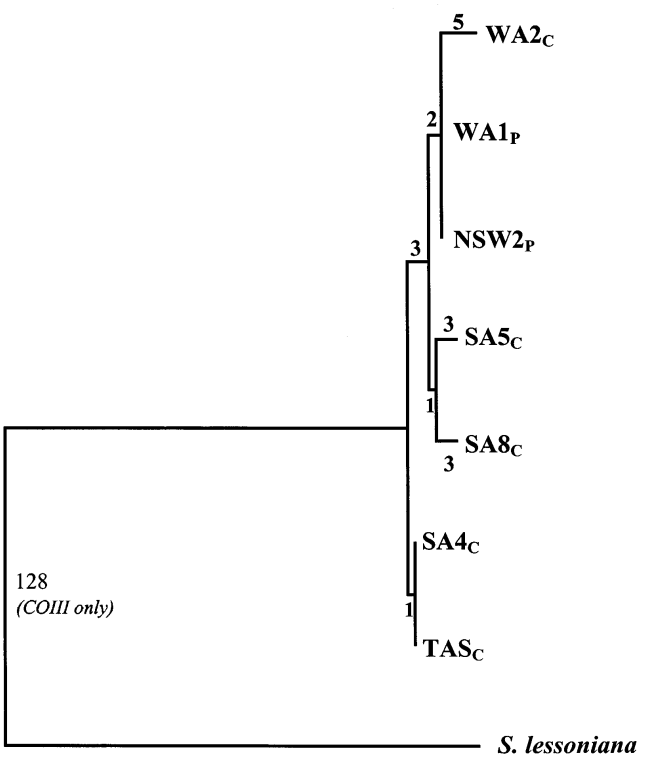

Fig. 3. Sepioteuthis australis. Maximun parsimony tree for 2 peripheral (p) and 5 central (c) calamary for the COII and COIII sequence data. Sample abbreviations as in Fig. 1 totally infertile to maintain the integrity of the 2 diagnostic loci. Our assessment here is limited to 1 simplistic measure of reproductive potential, namely the number of individuals that display the overall morphological features of sexual maturity. Given that an individual that appears sexually mature may still nevertheless be infertile, such a measure would be expected to underestimate the extent of any hybrid infertility if it existed. An examination of the reproductive status of hybrids indicates that they are indeed significantly less sexually mature $(p<0.0001)$ than the other 2 types $(p>$ 0.05 for peripheral vs central), although a moderate proportion (39 of 88) still showed signs of maturity (Table 6). This is despite hybrids being, on average, much larger than either parental taxon.

\section{Morphological distinctiveness a posteriori}

Because teeth counts on different suckers from the same calamary showed some minor variation, an aver- 
Table 6. Sepioteuthis australis. Results of a priori examination of 456 southern calamary in relation to genetic type. DML: dorsal mantle length

\begin{tabular}{|lccc|}
\hline Parameter & \multicolumn{2}{c|}{ Southern calamary genetic type } \\
& Central & Hybrid \\
\hline No. of immature females (Stages I-III) & 4 & 22 & 5 \\
No. of immature males (Stages I-III) & 5 & 53 & 44 \\
No. of mature females (Stages IV-VI) & 22 & 55 & 11 \\
No. of mature males (Stages IV-VI) & 37 & 170 & 28 \\
Proportion of females & $26 / 68(38.3 \%)$ & $77 / 300(25.7 \%)$ & $16 / 88(18.2 \%)$ \\
Proportion immature & $9 / 68(13.3 \%)$ & $75 / 300(25.0 \%)$ & $49 / 88(55.7 \%)$ \\
DML at which 50\% of females mature & $148 \mathrm{~mm}$ & $150.4 \mathrm{~mm}$ & $206 \mathrm{~mm}$ \\
DML at which 50\% of males mature & $148 \mathrm{~mm}$ & $103.7 \mathrm{~mm}$ & $340.8 \mathrm{~mm}$ \\
& & & \\
\hline
\end{tabular}

age of both teeth counts and sucker diameter was obtained for each individual calamary. A clear relationship was found between DML and average sucker diameter (Fig. 4A). In marked contrast, no discernible relationship was found between average teeth counts and DML, indicating that teeth counts were not sizedependent (Fig. 4B). No significant differences were found in average teeth counts between sexes or sites within genetic type $(\mathrm{p}=0.86)$. There was, however, significant variability in the counts between genetic types $(p<0.001)$. The peripherals generally had fewer teeth than the centrals, while the hybrid counts were mostly intermediate. This relationship between average teeth counts and genetic type for the 133 calamary is shown in Fig. 5. The minor variation displayed amongst teeth counts from the same individual is insignificant against the backdrop of the differences between the 3 genetic types, and a single count per calamary provides the same level of discrimination.

\section{Distribution of the 3 forms}

An examination of the relative abundance of peripheral, central and $F_{1}$ individuals in regions of overlap provides additional insight into the 2 competing hypotheses. One the one hand, the proportion of hybrids in any one sample set does not differ significantly from that predicted for random mating under Hardy-Weinberg expectations (raw data from Table 1), a result entirely consistent with linkage (but not inconsistent with 2 taxa hybridizing at random). In contrast, the proportions of peripheral, central and $F_{1}$ individuals differ significantly from each other in different years when the 2 sets of temporal replicates are compared (Samples WA2a vs WA2b, $\mathrm{p}=0.001$; Samples SA4a vs SA4b, $p=0.013$; raw data from Table 1). This is an unlikely outcome for simple linkage within a stable subpopulation in the absence of strong selection, and implies the existence of 2 taxa whose relative abundance in regions of overlap can vary markedly between catch efforts. In conclusion, the available evidence supports the notion that the southern calamary comprises 2 taxa with partially overlapping distributions and which hybridize at random in zones of overlap. Given this, the allozyme data have been analysed separately for each taxon (Tables $3 \& 4$ ).
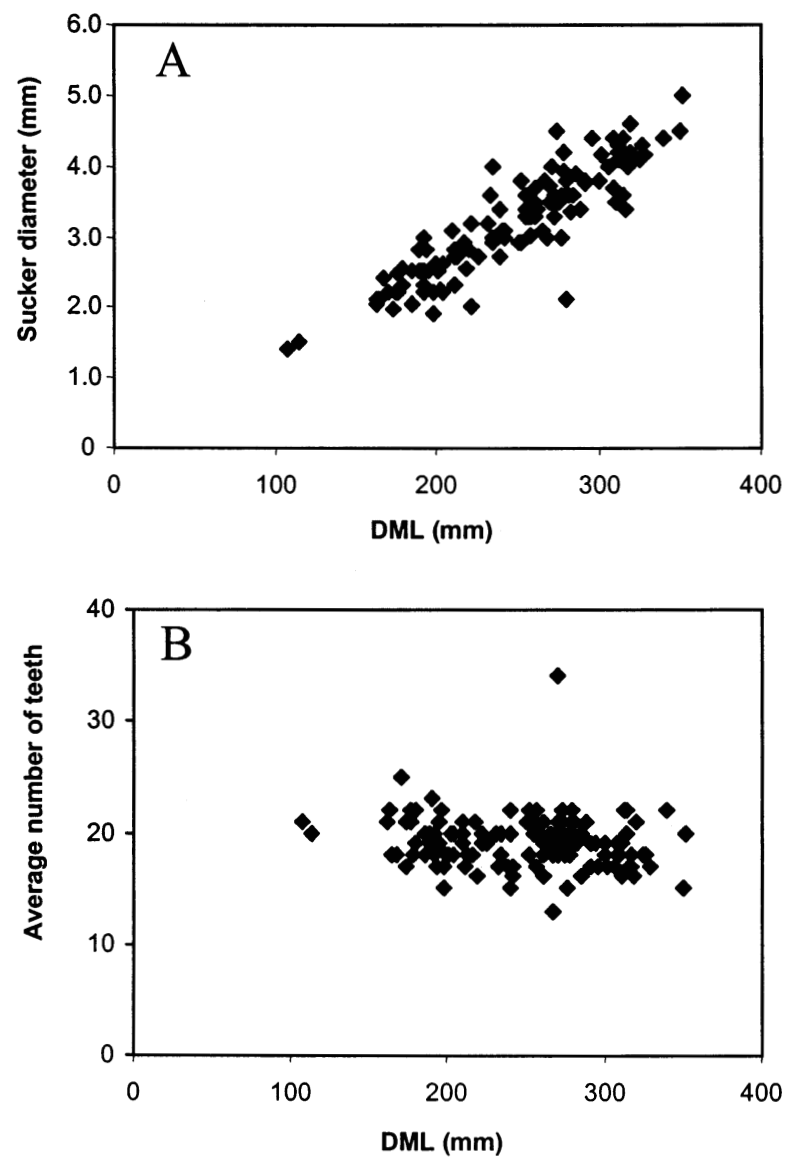

Fig. 4. Sepioteuthis australis. Sucker growth. Relationship between dorsal mantle length (DML) and (A) average sucker diameter and (B) average teeth counts 


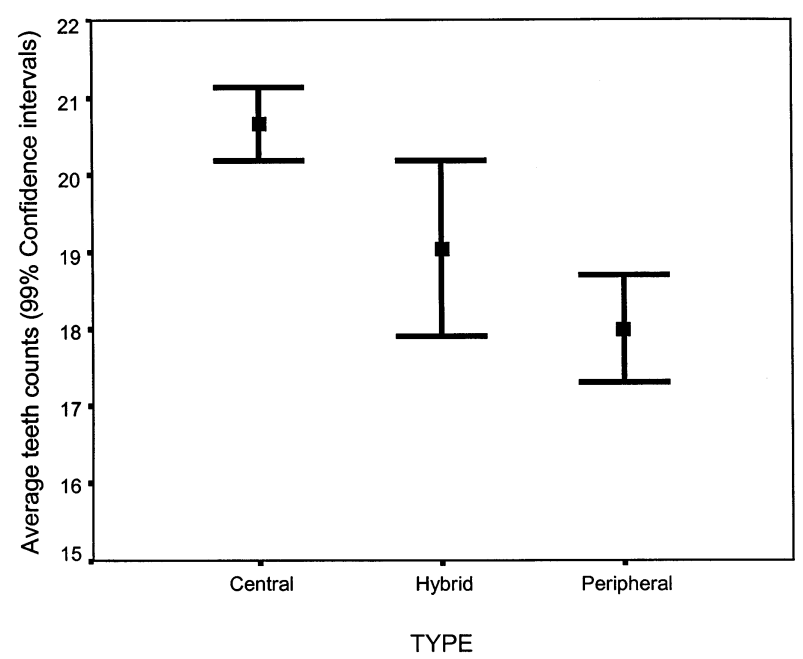

Fig. 5. Sepioteuthis australis. Mean $\pm 99 \%$ confidence intervals of teeth counts for the 3 genetic types of southern calamary from Western Australia and South Australia

\section{Population structure within the peripheral form}

The allozyme data (summarised in Table 3) for the peripheral form of Sepioteuthis australis were analysed for a suite of population genetic measures available in the program GENEPOP. The measures examined were (1) deviation from Hardy-Weinberg expectations at each locus in each sample set (testing for both a deficit and an excess of heterozygotes), (2) linkage disequilibrium between each locus in each sample set, and (3) genic differentiation between pairwise comparisons of sample sets at each locus. In all cases the 5 individuals from South Australia (at Sites SA3, SA4, and SA6) were pooled into a single sample set ('SA $A_{P}$ ') The replicate sample sets at Albany (WA2 $\mathrm{a}_{\mathrm{P}}$ and $W A 2 b_{P}$ ) were initially treated as separate, and subsequently pooled after failing to show significant differences in any measure.
There were no significant deviations from HardyWeinberg expectations at any locus when testing for heterozygote excess, and only one significant outcome testing for heterozygote deficiency ( $N p 1$ in sample set NSW2 $2_{\mathrm{P}}, 0.01<\mathrm{p}<0.05$ ). No evidence of linkage between any 2 loci was found in any sample set. Pairwise comparisons of allele frequency indicate the presence of 3 discrete stocks in the peripheral form, characterised by significant differences at $\geq 1$ of 4 loci (Table 7). These are referred to hereafter as the New Zealand (NZ), eastern and western stocks. The most distinctive sample set is that from New Zealand, distinguishable at 2 to 4 loci from all other sample sets, even including those with small sample sizes (' $\mathrm{SA}_{\mathrm{P}}$ ' and Site NSW1; both $\mathrm{n}=5$ ). Amongst the Australian sample sets, the eastern 2 from Eden (NSW1 $1_{\mathrm{P}}$ ) and Newcastle $\left(\mathrm{NSW} 2_{\mathrm{P}}\right.$ ) are distinguishable from most others at PepD. The single exception involves the small ' $\mathrm{SA}_{\mathrm{P}}$ ' sample set; a qualitative examination of the allozyme frequency data (Table 3) reveals that the SA individuals appear to belong with the western stock.

Estimates of genetic divergence (Nei's genetic distance, $D$ ) between sample sets range from 0.000 to 0.001 within the western stock, and 0.001 within the eastern stock. The eastern and western stocks differ on average at 0.008 (Nei's $D$; Fig. 6), whilst both differ from the New Zealand stock at much greater levels (eastern vs New Zealand, average $D=0.027$; western vs New Zealand, average $D=0.026$ ). There were insufficient sample sets within any stock to attempt an isolation-by-distance analysis.

The F-statistics for all 8 sample sets support the assertion that there is significant genetic divergence between New Zealand and Australia (Wright's fixation index, $F_{S T}=0.155 ; 95 \%$ confidence intervals 0.0307 to 0.245), but no deviation from panmixia within sample sets (inbreeding coefficient, $F_{I S}=0.031 ; 95 \%$ confidence intervals $=0.047$ to 0.137 ). Both values are nonsignificant when the New Zealand sample set is removed.

Table 7. Sepioteuthis australis. Summary of pairwise comparisons of allele frequency between sample sets for peripheral calamary. Below diagonal: number of significant differences where $\mathrm{p}<0.05$; above diagonal: loci involved. ${ }^{*} 0.01<\mathrm{p}<0.05$;

${ }^{* *} 0.001<\mathrm{p}<0.01 ;{ }^{* * *} \mathrm{p}<0.001$; number of haploid genomes for each sample set in parentheses; all $\mathrm{p}$ values are corrected

\begin{tabular}{|c|c|c|c|c|c|c|c|}
\hline Sample set & $\begin{array}{l}\text { WA1 } 1_{p} \\
(100)\end{array}$ & $\begin{array}{c}\text { WA2 }_{p} \\
(82)\end{array}$ & $\begin{array}{c}\text { WA } 3_{p} \\
(22)\end{array}$ & $\begin{array}{l}\text { 'SA }{ }_{p}^{\prime} \\
(10)\end{array}$ & $\begin{array}{l}\mathrm{NSW}_{\mathrm{p}} \\
\quad(10)\end{array}$ & $\begin{array}{l}\mathrm{NSW}_{\mathrm{p}} \\
(96)\end{array}$ & $\begin{array}{l}\mathrm{NZ}_{\mathrm{p}} \\
(42)\end{array}$ \\
\hline WA $1_{p}$ & - & & & & $P e p D^{*}$ & $\operatorname{Pep} D^{* * *}$ & $\operatorname{Dia}^{* * *}, \operatorname{Pep} D^{* * *}, P g k^{* * *}$ \\
\hline WA $2_{\mathrm{p}}$ & 0 & - & & & PepD* & $\operatorname{Pep} D^{* * *}$ & $\operatorname{Dia}^{* * *}, N_{p} 1^{*}, P e p D^{* * *}, P g k^{* * *}$ \\
\hline WA $3_{\mathrm{p}}$ & 0 & 0 & - & & $P e p D^{*}$ & $P e p D^{* * *}$ & $\operatorname{Dia}^{* * *}, \operatorname{Pep} D^{* * *}$ \\
\hline${ }^{\prime} \mathrm{SA}_{\mathrm{p}}{ }^{\prime}$ & 0 & 0 & 0 & - & & $P e p D^{* * *}$ & $\operatorname{Dia}^{* * *}, \operatorname{Pep} D^{* * *}$ \\
\hline NSW $1_{p}$ & 1 & 1 & 1 & 0 & - & & $\operatorname{Dia}^{* * *}, P g k^{*}$ \\
\hline NSW2 $2_{p}$ & 1 & 1 & 1 & 1 & 0 & - & $\operatorname{Dia}^{* * *}, N p 1^{* * *}, P g k^{*}$ \\
\hline $\mathrm{NZ}_{\mathrm{p}}$ & 3 & 4 & 2 & 2 & 2 & 3 & - \\
\hline
\end{tabular}




\section{Population structure within the central form}

The allozyme data for the central form of Sepioteuthis australis (summarised in Table 4) were analysed for the same array of population genetic parameters, plus the additional calculation of $F_{S T}$ values between sample sets for an isolation-by-distance analysis. In all cases, the 11 Western Australian calamary (WA2a, WA2b, and WA3) were pooled into a single sample set $\left(\mathrm{WA}_{\mathrm{C}}\right)$. The replicate samples from Pearson Island $\left(\mathrm{SA} 4 \mathrm{a}_{\mathrm{C}}\right.$ and $\left.\mathrm{SA} 4 \mathrm{~b}_{\mathrm{C}}\right)$ and Tasmania $\left(\mathrm{TASa}_{\mathrm{C}}\right.$ and $\mathrm{TASb}_{\mathrm{C}}$ ) were initially treated as separate sample sets, and subsequently pooled after failing to reveal significant differences at any parameter. Consisting as it did of only a single individual, the New Zealand sample set could not be used 'as is' in any analysis. To partially circumvent this problem, the assessment of genic differentiation was carried out using a composite New Zealand gene pool ( $\mathrm{n}=14$ haploid genomes) consisting of the single pure central calamary plus one allozyme from each of the 12 hybrids (only where there was no ambiguity as to what allozyme would have been derived from any putative central parent).

There was no evidence of deviation from HardyWeinberg expectations (involving either heterozygote deficiency or excess) at any locus, and no indication of linkage disequilibrium between loci within any sample set. Pairwise comparisons of allele frequencies revealed minor evidence of stock structure within the central form. The composite $\mathrm{NZ}_{\mathrm{C}}$ sample set appeared to represent a distinct stock, displaying significant allelic differences from all Australian sample sets at Dia (all $\mathrm{p}<0.001$ except for $\mathrm{SA} 2_{\mathrm{C}}, \mathrm{SA} 3_{\mathrm{C}}, \mathrm{SA} 5_{\mathrm{C}}$, and SA6 $6_{C}$, where $\mathrm{p}<0.01$, and $\mathrm{SA} 7_{\mathrm{C}}$, where $\mathrm{p}<0.05$ ). There was no indication of further substructuring within the Australian stock.

The Australian and New Zealand stocks (as represented by a single specimen) differed at an average Nei's $D$ of 0.007 (range 0.005 to 0.013 ; Fig. 6). Sample sets within the Australian stock exhibited levels of genetic divergence ranging between 0.000 and 0.003 (Nei's $D$ ) from each other. An analysis comparing pairwise $F_{S T}$ values against geographic distance (Fig. 7) revealed no compelling evidence that an isolation-bydistance model is appropriate for these data. The $F$-statistics for all 13 sample sets also provide weak support for the existence of a separate stock in New Zealand. A marginally significant positive value for $F_{S T}$ $\left(F_{S T}=0.0087 ; 95 \%\right.$ confidence intervals 0.0188 to $0.0001)$ becomes non-significant after removing the NZ sample set $\left(F_{S T}=0 ; 95 \%\right.$ confidence intervals 0.0014 to -0.0041). $F_{I S}$ values for both analyses do not differ significantly from zero, supporting the assumption of panmixia within sample sets.

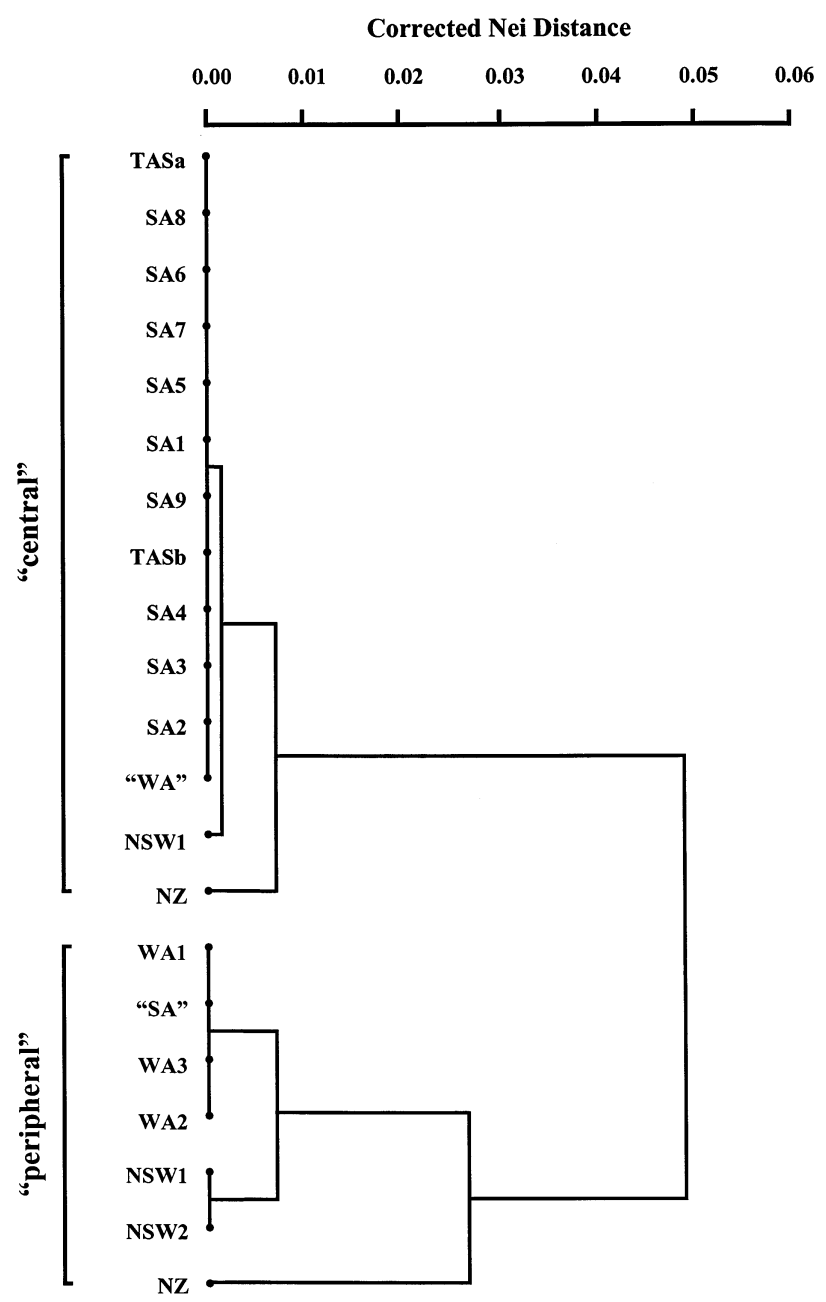

Fig. 6. UPGMA Dendrogram based on Nei's genetic distance for all peripheral and central sample sets

\section{Comparisons of peripheral, central, and hybrid forms in overlap zones}

An examination of Tables 3 to 5 reveals that peripheral, central and hybrid sample sets display similar allozyme frequencies in zones of overlap at all loci except the 2 diagnostic markers. Although sample sizes were not always adequate for rigorous statistical analysis, pairwise comparisons of peripheral, central, and hybrid allele frequencies within initial sample sets did not reveal any significant differences at the nondiagnostic loci. Such an outcome is most notable for the New Zealand sample sets (using the composite central/hybrid data to represent the central form). For both the peripheral and central forms, the New Zealand stock is distinguishable from some or all Australian sample sets by correlated differences in allele frequency at the markers Dia, Np1, and Pgk, and yet $\mathrm{NZ}_{\mathrm{P}}$ and $\mathrm{NZ}_{\mathrm{C}}$ do not differ significantly from each other at 


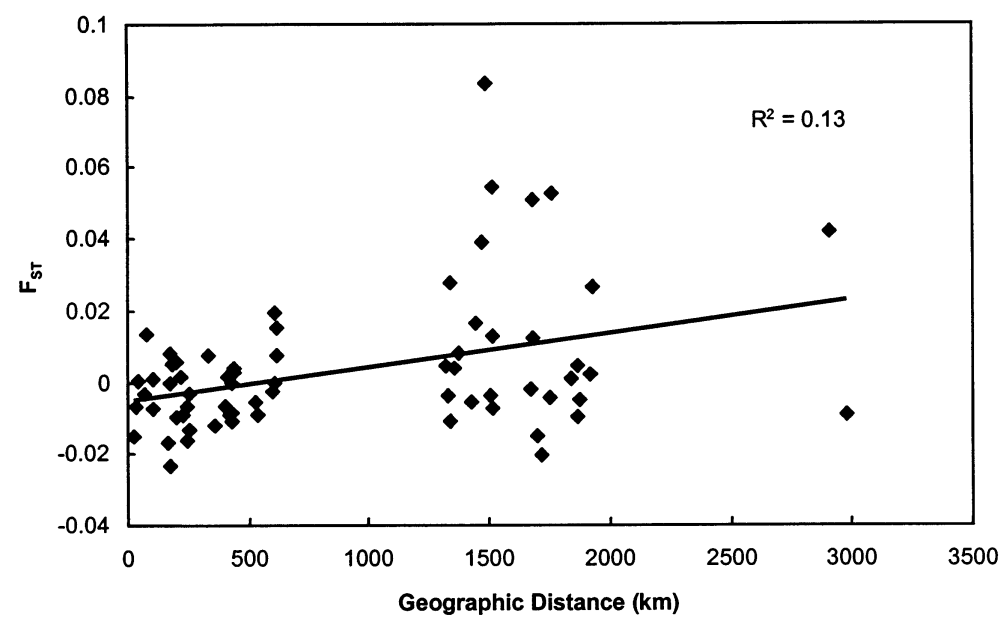

Fig. 7. Sepioteuthis australis. Isolation-by-distance analysis for the Australian sample sets of the central form (plot of Wright's fixation index $F_{S T}$ vs geographic distance)

any locus other than PepD and Fdp, the markers diagnostic for the 2 taxa. Given that 2 Australian stocks of the peripheral form are distinguishable (based on major differences in the frequency of alleles $a+b+c+d$ at $P e p D$; Tables $3 \& 7$ ), it is worth noting that hybrid calamary from most sample sets (Table 5) bear the genetic signature of the co-existing peripheral stock rather than of the alternate peripheral stock. Thus, distinctive western and eastern 'stocks' of hybrids can be recognized (Sites SA8, TAS, and NSW2 were not able to be assigned because of small sample sizes) based on their PepD profile.

\section{DISCUSSION}

\section{Systematic status of the 3 genetic forms in Sepioteuthis australis}

The allozyme data presented herein demonstrate complete linkage disequilibrium between $F d p$ and PepD amongst 757 southern calamary examined across a geographic range of more than $5500 \mathrm{~km}$. This despite no indication of heterozygote deficiency in any sample set at either locus, nor, with one exception, at any other locus. Two obvious hypotheses can be envisaged to account for this extraordinary pattern: (1) the 2 loci are very tightly linked, or (2) there are 2 taxa within Sepioteuthis australis, diagnosable by fixed differences at $F d p$ and $P e p D$, which mate at random wherever they co-exist, but where the hybrids are infertile.

The available morphological and reproductive data largely support the second hypothesis, although they do not specifically exclude the first. Southern calamary belonging to the peripheral and central forms were distinguishable from each other using the simple morphological feature of the number of teeth on the largest sucker(s) of the largest tentacle(s). They also show significant differences in growth rates after adjusting for sex and site (L.T. unpubl. data). These findings are only consistent with the hypothesis of linkage if genetic control of both these characters also resides within or adjacent to the $F d p / P e p D$ linkage group. Furthermore, hybrid calamary differed significantly from both putative parental forms in 4 biological parameters; sex ratio, proportion of sexuallymature individuals, dorsal mantle length at maturity (present study), and growth rates (L.T. unpubl. data). If linkage alone is involved, these characters too must presumably in some way be associated with the $F d p / P e p D$ linkage group.

The existence of 3 diagnostic characters and other biological correlates in sympatry would ordinarily be sufficient to warrant the recognition of 2 species within Sepioteuthis australis. However, the common occurrence of calamary with the genetic profiles of $F_{1}$ hybrids, whose abundance can be predicted under expectations of random mating between the species, creates some uncertainty about such a conclusion. Thus, it is important to explore in more detail the alternative scenarios under which such a result could be obtained within a single species. Pronounced linkage disequilibrium in natural populations between unrelated genes (i.e. not members of a gene family) can occur when the genes concerned are (1) very tightly linked, (2) associated with a polymorphism for some structural chromosomal alteration (usually an inversion or reciprocal translocation, both of which suppress recombination during meiosis in a heterozygote), or (3) not subjected to recombination due to some other mechanism which either suppresses crossing-over or does not allow recombinants to survive (Hartl \& Jones 1998). Can any of these situations be operating in the southern calamary?

Explanation 1 is highly implausible in this instance. Firstly, 2 of 13 randomly selected polymorphic markers would have to be so tightly linked that recombinants have never been recovered over successive generations. Loci showing as little as $\sim 1 \%$ recombination are usually close to linkage equilibrium in natural populations, and even adjacent loci usually show some evidence of recombination over moderate periods of evolutionary time (Maynard Smith 1994). In addition, a minimum of 2 other genetic makers (i.e. increasing the proportion of 'linked' markers to $\sim 25 \%$ ), one influencing sucker teeth and the other 
growth rates, would also have to be part of the same tight linkage group.

Explanation 2 is also unlikely given the available information on squid karyotypes. Although the karyotype of the southern calamary is not known, it can be reasonably inferred from studies of other species of squid across 5 genera and 2 families. Two sepioids and 3 other loliginids, including the northern calamary Sepioteuthis lessoniana, all possess a karyotype of $2 \mathrm{~N} \sim$ 92 , with no individual chromosome larger than $4 \%$ nor smaller than $1.1 \%$ of total karyotype length (Gao \& Natsukari 1990). Given such evolutionary conservatism, $S$. australis is unlikely to differ greatly from this general pattern. Thus, even if 2 entire chromosomes were involved in a reciprocal translocation, linkage disequilibrium would still only be evident for at most $8 \%$ of loci examined. Polymorphism for multiple chromosomal rearrangements, such as is found associated with semi-sterility in some plants (Hartl \& Jones 1998) would need to be operating to generate the results obtained here within a single species. Moreover, even where inversion or translocation polymorphisms are evident within a species, there is rarely complete linkage disequilibrium between included loci, due to phenomena such as double cross-overs, gene conversion and 'middle-gene' effects (Loukas et al. 1979, Krimbas \& Powell 1992).

For similar reasons, Explanation 3 is also unlikely to be a factor for these data. Assuming a karyotype of $2 \mathrm{~N} \sim 92$, any suppression of crossing-over or recombination would need to operate simultaneously and concertedly on a number of different chromosomes to generate pronounced linkage disequilibrium amongst at least $25 \%$ of loci sampled. Some species are known in which recombination does not occur within individuals, but these instances only occur in one sex (Maynard Smith 1994). Absence of recombination would need to feature in both sexes of Sepioteuthis australis for this explanation to be tenable and, if present, might also be expected to lead to many other instances of linkage disequilibrium.

Thus we argue that the results obtained here for southern calamary are inconsistent with the hypothesis of a single species, and instead suggest the existence of 2 taxa, capable of hybridizing freely in areas of overlap. Nevertheless, such a biological scenario does not preclude the existence of chromosomal differences between these 2 taxa. Closely-related taxa differing in structural chromosomal features such as translocations or inversions often exhibit partial or total reproductive isolation (White 1978). Any hybrids produced may experience difficulties in chromosomal pairing and proper disjunction during meiosis, resulting in partial or complete sterility (Avise 1994). And, while total sterility would maintain the genetic integrity of both taxa (in this case species), partial sterility would result in some degree of genetic exchange between the 2 gene pools for all loci not included within the structural rearrangement distinguishing the 2 taxa. As a consequence, allele frequencies at most loci would tend to converge on intermediate levels in both taxa, the extent of introgression depending on the degree of sterility encountered in hybrids and the effects of selection.

Such an explanation would be consistent with 2 of the major outcomes of this study, namely (1) only $F_{1}$ hybrids were found, and (2) allele frequencies at loci other than Fdp and PepD did not differ significantly between central and peripheral individuals in any zone of overlap, including New Zealand (where both the $\mathrm{NZ}_{\mathrm{P}}$ and by inference the $\mathrm{NZ}_{\mathrm{C}}$ stocks were diagnosable from their eastern counterparts but not from each other). Hybrids could still be partially fertile, but would only produce gametes with parental genotypes at loci located within the chromosomal rearrangement (Fdp and PepD in this instance). Loci not linked to the rearrangement would participate in some level of genetic exchange such that in any one site the 2 'parental' taxa would converge on similar allelic profiles at all non-linked loci. As a consequence, each of the 'peripheral', 'central' and 'hybrid' genetic types would actually harbour a small percentage of calamary of hybrid ancestry $\left(F_{1} \times\right.$ peripheral, $F_{1} \times$ central, $F_{2}$ etc. $)$, depending upon the fertility of each type of secondgeneration cross. Nevertheless, partial sterility in the hybrids would still ensure that other genetic differences would be maintained at loci under the direct influence of selection.

Unfortunately, the data presented herein cannot resolve whether there is introgression between the 2 taxa. Our limited data plus those of Triantafillos (unpubl. data) suggest that hybrids, whilst more vigorous than either parental form, are at best semi-sterile. Hybrid calamary were, in general, substantially larger than mature peripheral and central calamary, yet a majority were sexually immature, as assessed by a macroscopic examination of the gonads. Interestingly, the only site where mature hybrids were commonly found was New Zealand. Despite having fully formed reproductive organs, these hybrids may still not be able to produce reproductively competent offspring. Galbreath \& Thorgaad (1995) found that seemingly mature hybrid salmon produced offspring that never progressed past $30 \mathrm{~d}$, and Lincoln (1981) found that male triploid plaice crossed with flounder produced hybrids that seemed sexually mature, but that only produced sterile gametes.

We hesitate to apply the term 'species' to these 2 taxa until further investigation has addressed the reproductive potential of hybrids. As such, our data do not allow 
us to draw conclusions about the nomenclature of Sepioteuthis spp. in Australia and New Zealand. The allozyme data do offer general support for the conclusions of Lu \& Tait (1983) that the same species of Sepioteuthis occurs in both Australia and New Zealand. However, if hybrids turn out to be largely infertile (and therefore both peripheral and central forms are good biological species), the name ' $S$. bilineata' may nevertheless still apply to 1 of the 2 species in New Zealand. Such ambiguity could then only be resolved by a reexamination of holotypes for sucker-teeth counts and any other diagnostic markers that may emerge in the future.

Unlike the morphological and the growth data, the pilot mtDNA data did not fully support the hypothesis of 2 taxa. Several explanations are possible here. As closely related taxa often do not display reciprocal monophyly for their mitochondrial gene trees (Avise 1994), the results of our pilot analysis may reflect the true mitochondrial phylogeny. Alternatively, the protein-encoding genes chosen may be too conservative to reveal any fine-scale phylogenetic structure. Low levels of genetic diversity have been found in other loliginids such as Loligo pealei (Garthwaite et al. 1989), and may be characteristic of squid in general (Ally \& Keck 1978, Brierley et al. 1993). A third possibility is that the mtDNA data are informative in that they identify a clade comprising the 2 'peripheral' calmary (identical sequences despite being from opposite ends of the continent) plus a 'central' calmary from Albany, a region where hybrids are commonly encountered but where the central form is rare. Such a finding may actually reflect the existence of some introgression, with the Albany individual displaying a peripheral mtDNA haplotype originally derived from its original maternal parent. However, any discussion of introgression must remain pure speculation given the low levels of diversity and the absence of further sequence data.

\section{Population structure within each genetic group}

Regardless of the extent of introgression occurring between peripheral and central forms of the southern calamary, the existence of these 2 taxa and their hybrids requires that all 3 genetic groups be assessed separately for any evidence of population structure. Unfortunately, all 3 analyses suffer from the same deficiency, namely inadequate sample size. Few sample sets conform to our a priori goal of $n=50$ ( 2 of 10 peripheral, 6 of 17 central, 0 of 14 hybrid) and this severely limits the ability of the allozyme data to probe too deeply into population structure. This limitation is further compounded by the high probability of a Type II error accompanying all allozyme analyses of popula- tion structure (Richardson et al. 1986). Nevertheless, it is possible to make some statistically rigorous observations regarding the minimum number of stocks present within each genetic form.

\section{Peripheral form}

Three subpopulations were shown to exist within the peripheral form, one each in Western Australia, southeast Australia and New Zealand. Multi-locus heterogeneity between the Australian and New Zealand stocks rules out the possibility of a locus-specific rather than population-structure effect (Richardson et al. 1986). It is not surprising to find genetic differences between mainland Australia and New Zealand samples, because these 2 regions are separated by $>2000 \mathrm{~km}$ of open ocean, much of which is of considerable depth $(>2000 \mathrm{~m})$. Regular migrations and subsequent genetic exchange across such a geographic barrier by a neritic squid are unlikely (see Brierley et al. 1995). The eastern and western stocks of Australia are diagnosable only at 1 locus $(P e p D)$, albeit involving major clear differences in allele frequency. These 2 stocks are also, to a certain extent, oceanographically isolated. The eastern stock is kept more or less isolated from the western stock by the East Australian Current (Boland \& Church 1981).

\section{Central form}

In stark contrast to the population heterogeneity observed within the peripheral type, very little population substructuring was detected within the central form. Allele frequencies from Tasmania were similar at all loci to those found in Western Australia, a distance of nearly $3000 \mathrm{~km}$. These data are consistent with a single, randomly-mating stock across Australia. As with the peripheral form, there were some significant allelic differences between Australia and New Zealand, suggesting that the New Zealand sample was a separate stock.

Prior to this study, the only information concerning the stock structure in southern calamary was gained from 2 short-term tagging studies (Smith 1983, Triantafillos 1998). Both these studies showed that although most individuals are predominantly sedentary, the species is capable of long and fairly rapid migrations. Such mobility would potentially facilitate gene flow over considerable distances. In the absence of strong selection pressures, the effective number of migrants between regions that is necessary to prevent genetic drift from producing significantly different allele frequencies is small, i.e. 1 to 10 individuals per generation (Allendorf et al. 1987). Given that large 
stretches of the southern Australian coast are suitable habitat for calamary, it is feasible that there is sufficient movement of individuals from one region to the next, in a stepping-stone fashion, to prevent subpopulations emerging within the entire Australian central stock.

\section{Hybrid form}

Three stocks of the hybrid form of Sepioteuthis australis are broadly identifiable from the allozyme data, based on the distribution of parental stocks and from an examination of allozyme frequencies (Table 5). The western and eastern hybrid stocks differ only at one marker $(P e p D)$, whilst the New Zealand hybrid stock is diagnosable from the 2 Australian stocks using the same loci that distinguish both New Zealand central and New Zealand peripheral stocks from their respective Australian counterparts (Dia, Pgk and/or PepD). Although the rarest overall, hybrid calamary were the most abundant of the 3 genetic groups in 2 of the 19 sample sets (Albany 96 and Esperance), demonstrating that they are likely to constitute a major part of the catch in the major zones of overlap, i.e. (1) the south coast of WA through to the west coast of SA, (2) the south-east coast of Australia, and (3) at least 1 region in New Zealand. As such, they need to be considered as part of any management plan for the southern calamary fishery.

\section{Biogeographic regions}

The geographic distributions of the 2 taxa are closely related to the biogeographical regions proposed by Knox (1963). The distribution of the central type coincides with the Flindersian and Maugean Provinces postulated by Womersley \& Edmonds (1958), which have affinities intermediate between cool- and warmtemperate regions. In comparison, the peripheral type prefers the warmer waters of the warm temperate regions of the Western Australian, Peronia and Auporian Provinces found in Western Australia, New South Wales, and New Zealand, respectively. Most of the hybrids were found in the transitional zones between these water masses. Such affinities to different oceanographic masses could account for the genetic divergence between the 2 taxa. For example, an allozyme study of the ommastrephid squid Nototodarus gouldii around New Zealand also revealed an allopatric sibling species, N. sloanii (Smith et al. 1981). The distributions of these 2 species were divided by the Substructuring Tropical Convergence Zone (Smith et al. 1981). It may be that under normal conditions the peripheral and central forms of Sepioteuthis australis may be largely allopatric, coming together only occasionally when prevailing oceanographic conditions allow (Brierley et al. 1993). For example, the assorted peripherals around the islands of southwestern South Australia may be attributable to the Leeuwin Current (e.g. Maxwell \& Cresswell 1981). This current carries warm water from northwestern Australia, where the peripherals dominate, through Albany, and then across the Great Australian Bight (Cresswell 1991). At its strongest, the Leeuwin Current eventually dissipates after it reaches the islands of southwestern South Australia (Cresswell 1991). This may explain why there are no peripherals east of these islands. Similarly, the East Australian Current probably controls the distribution of the peripherals on the eastern side of Australia and New Zealand. Gill (1997) suggested that many Australian marine reptiles were assisted or carried to New Zealand by the East Australian Current.

\section{Implications for management of the southern calamary fishery}

The results of our study have clear implications for the management of southern calamary. The existence of sibling species, and their unknown contribution to respective fisheries, would complicate markedly the construction of stock-assessment models and provisions of subsequent fishery advice (Beddington et al. 1990), especially as part of the observed variance in reproductive, size and age-related parameters are species-specific. At present, southern calamary in Australia are managed as if all individuals were members of a single, interbreeding stock. The results presented here indicate a minimum of 5 stocks, namely (1) western peripheral, (2) eastern peripheral, (3) central, (4) western hybrids, and (5) eastern hybrids. Three additional stocks occur in New Zealand: NZ peripheral, NZ central, and NZ hybrids. With the exception of Perth, Port Lincoln, Myponga Beach and Kangaroo Island, all sites had more than 1 genetic type present. At 6 localities, all 3 genetic types were present at various frequencies (Albany, Esperance, Flinders Island, Pearson Island, Eden, and New Zealand). Moreover, both temporal replicates revealed significant differences in the proportions of the 3 stocks taken at random. This result may reflect genuine temporal differences in abundance, or alternatively the differential sampling of taxon-specific differences in the microhabitat. Re-gardless of the explanation, where stocks differ in their relative strengths in space and time, none can be harvested at an optimal level, since either the weaker stocks are over-exploited or the stronger ones remain under-exploited (Ryman \& Utter 1987). 


\section{Concluding remarks}

The results of this study and the occurrence of cryptic speciation in yet another species of loliginid squid reinforces the need for a systematic molecular assessment of the target 'species' as a first step in any study on the biology of squid. Ambiguity of this sort reiterates the relevance of systematics and the importance of unequivocally identifying whether the populations under study are conspecific before undertaking any assessment of population structure. In the case of Sepioteuthis australis, the present study casts doubts over the validity of all previous ecological work, the results of which were not interpreted according to the true systematic complexity present.

Acknowledgements. This research was funded by the South Australian Marine Scalefish Integrated Management Committee and the Northern Territory University. We thank the staff of the Evolutionary Biology Unit (South Australian Museum) for their technical help and constructive criticisms of the manuscript. We are grateful to David Short for his tireless help in collecting many of the southern calamary. We also grateful to the crew of the RV 'Ngerin' for their help in collecting southern calamary, and Bruce Bird of Moana Pacific Fisheries Ltd for generously supplying southern calamary from New Zealand. Financial support was obtained by the senior author from a postgraduate scholarship from the Northern Territory University.

\section{LITERATURE CITED}

Adams M, Baverstock PR, Watts CHS, Reardon T (1987) Electrophoretic resolution of species boundaries in Australian Microchiroptera. I. Eptesicus (Chiroptera, Vespertilionidae). Aust J Biol Sci 40:143-62

Allendorf FW, Ryman N, Utter FM (1987) Genetics and fishery management. Past, present and future. In: Ryman N, Utter FM (eds) Population genetics and fishery management. University of Washington Press, Seattle, p 1-18

Ally JRR, Keck SC (1978) A biochemical genetic population structure study of the market squid, Loligo opalescens, along the California coast. Calif Fish Game 169:113-121

Augustyn CJ, Grant WS (1988) Biochemical and morphological systematics of Loligo vulgaris vulgaris Lamarck and Loligo vulgaris reynaudii d'Orbigny nov. comb. (Cephalopoda: Myopsida). Malacologia 29:215-233

Avise JC (1994) Molecular markers, natural history and evolution. Chapman \& Hall, New York

Beddington JR, Rosenberg AA, Crombie JA, Kirkwood GP (1990) Stock assessment and the provision of management advice for the short fin squid fishery in Falkland Islands. Fish Res 8:351-365

Boland FM, Church JA (1981) The East Australian Current, 1978. Deep-Sea Res 28A:937-957

Bonnaud L, Boucher-Rodoni R, Monnerot M (1997) Phylogeny of decapod cephalopods inferred from mitochondrial DNA sequences. Mol Phylogenet Evol 7:44-54

Brierley AS, Rodhouse PG, Thorpe JP, Clarke MR (1993) Genetic evidence of population heterogeneity and cryptic speciation in the ommastrephid squid Martiala hyadesi from the Patagonian Shelf and Antarctic Polar Frontal Zone. Mar Biol 116:593-602

Brierley AS, Thorpe JP, Pierce GJ, Clarke MR, Boyle PR (1995) Genetic variation in the neritic squid Loligo forbesi (Myopsida: Loliginidae) in the northeast Atlantic Ocean. Mar Biol 122:79-86

Coleman N (1984) Molluscs from the diets of commercially exploited fish off the coast of Victoria, Australia. J Malac Soc Aust 6:143-154

Cresswell GR (1991) The Leeuwin Current-observations and recent models. J Proc R Soc West Aust 74:1-14

Galbreath PF, Thorgaad GH (1995) Sexual maturation and fertility of diploid and triploid Atlantic salmon $\times$ brown trout hybrids. Aquaculture 137:299-311

Gales R, Pemberton D, Lu CC, Clarke MR (1993) Cephalopod diet of the Australian fur seal: variation due to location, season and sample type. Aust J Mar Freshw Res 44: $657-671$

Gao Y, Natsukari Y (1990) Karyological studies on seven cephalopods. Venus, Kyoto 49:126-145

Garthwaite RL, Berg CJ Jr, Harrigan J (1989) Population genetics of the common squid Loligo pealei Le Seur, 1821, from Cape Cod to Cape Hatteras. Biol Bull 177:287-294

Gill BJ (1997) Records of turtles and sea snakes in New Zealand, 1837-1996. NZ J Mar Freshw Res 31:477-486

Hartl DL, Jones EW (1998) Genetics: principles and analysis, 4 th edn. Jones \& Bartlett, Sudbury, MA

Higgins DG, Bleasby AJ, Fuchs R (1992) Clustal V: improved software for multiple sequence alignment. Cabios 8: 189-191

Hillis DM, Mabel BK, Moritz C (1996) Applications of molecular systematics. In: Hillis DM, Moritz C, Mabel BK (eds) Molecular systematics. Sinauer Associates, Sunderland, MA, p 515-43

Ihssen PE, Brooke HE, Casselman JM, McGlade JM, Payne NR, Utter FM (1981) Stock identification: materials and methods. Can J Fish Aquat Sci 38:1838-1855

Izuka T, Segawa S, Okutani T (1996) Biochemical study of the population heterogeneity and distribution of the oval squid Sepioteuthis lessoniana complex in southwestern Japan. Am Malac Bull 12:129-135

Katugin ON (1995) Genetic differentiation in Berryteuthis magister from the North Pacific. ICES Mar Sci Symp 199: 459-467

Knox GA (1963) The biogeography and intertidal ecology of the Australian coasts. Oceanogr Mar Biol Annu Rev 1: 341-404

Krimbas C, Powell JR (1992) Introduction. In: Krimbas C, Powell JR (eds) Drosophila inversion polymorphisms. CRC Press, London, p 1-52

Kumar S, Tamura K, Nei M (1993) MEGA: molecular evolutionary genetics analysis. Version 1.0. The Pennsylvania State University, University Park, PA

Lincoln RF (1981) Sexual maturation in female triploid plaice, Pleuronectes platessa, and plaice $\times$ flounder, Platichthys flesus, hybrids. J Fish Biol 19:499-507

Lipinski M (1979) Universal maturity scale for the commercially important squids (Cephalopoda: Teuthoidae). The results of maturity classification of the Illex illecebrosus (LeSeur, 1821) populations for the years 1973-77. Res Docums Int Commn NW Atlant Fish (ICNAF) 79/11/38: $1-40$

Loukas M, Krimbas CB, Vergini Y (1979) The genetics of Drosophila subobscura populations. IX. Studies on linkage disequilibrium in four natural populations. Genetics 93: 497-523

Lu CC, Tait RW (1983) Taxonomic studies on Sepioteuthis 
Blainville (Cephalopoda: Loliginidae) from the Australian region. Proc R Soc Vict 95:181-204

Maxwell JGH, Cresswell GR (1981) Dispersal of tropical marine fauna to the Great Australian Bight by the Leeuwin Current. Aust J Mar Freshw Res 32:493-500

Maynard Smith J (1994) Evolutionary genetics. Oxford University Press, Oxford

Nei M (1978) Estimation of average heterozygosity and genetic distance from a number of individuals. Genetics 89:583-590

Raymond M, Rousset F (1995) GENEPOP. Version 1.2.: population genetics software for exact tests and ecumenicism. J Hered 86:248-249

Rice WR (1989) Analysing tables of statistical tests. Evolution 43:223-235

Richardson BJ, Baverstock PR, Adams M (1986) Allozyme electrophoresis: a handbook of animal systematics and population studies. Academic Press, New York

Ryman N, Utter F (1987) Population genetics and fishery management. Washington Sea Grant Program. University of Washington Press, Seattle, WA

Sambrook J, Fritsch EF, Maniatis T (1989) Molecular cloning: a laboratory manual, 2nd edn. Coldspring Harbor Laboratory Press, New York

Smith HK (1983) Southern calamary. Safic 7:11-17

Editorial responsibility: George Humphrey (Contributing Editor), Sydney, New South Wales, Australia
Smith PJ, Roberts PE, Hurst RJ (1981) Evidence for 2 species of arrow squid in the New Zealand fishery. NZ J Mar Freshw Res 15:247-253

Sokal RR, Sneath PHA (1963) Numerical taxonomy: the principles and practice of numerical classification. Freeman, San Francisco

Swofford DL (1993) PAUP: phylogenetic analysis using parsimony, Version 3.1. Illinois Natural History Survey, Champaign, IL

Triantafillos L (1997) Southern calamary. South Australia Fisheries Assessment Series No. 97/09. SARDI Aquatic Sciences, Adelaide

Triantafillos L (1998) Southern calamary. South Australia Fisheries Assessment Series No. 98/08. SARDI Aquatic Sciences, Adelaide

Weir BS (1990) Intraspecific differentiation. In: Hillis DM, Moritz CM (eds) Molecular systematics. Sinauer Associates, Sunderland, MA, p 373-410

White MJD (1978) Modes of speciation. WH Freeman \& Co, San Francisco

Womersely HBS, Edmonds SJ (1958) A general account of the intertidal ecology of South Australian Coasts. Aust J Mar Freshw Res 9:217

Yeatman J, Benzie JAH (1994) Genetic structure and distribution of Photololigo spp. in Australia. Mar Biol 118:79-87

Submitted: August 2, 1999; Accepted: February 21, 2000 Proofs received from author(s): February 26, 2001 